\title{
INVARIANT METRIC ON THE EXTENDED SIEGEL-JACOBI UPPER HALF SPACE
}

\author{
STEFAN BERCEANU
}

\begin{abstract}
The real Jacobi group $G_{n}^{J}(\mathbb{R})$, defined as the semidirect product of the Heisenberg group $\mathrm{H}_{n}(\mathbb{R})$ with the symplectic group $\operatorname{Sp}(n, \mathbb{R})$, admits a matrix embedding in $\operatorname{Sp}(n+1, \mathbb{R})$. The modified pre-Iwasawa decomposition of $\operatorname{Sp}(n, \mathbb{R})$ allows us to introduce a convenient coordinatization $S_{n}$ of $G_{n}^{J}(\mathbb{R})$, which for $G_{1}^{J}(\mathbb{R})$ coincides with the $S$-coordinates. Invariant one-forms on $G_{n}^{J}(\mathbb{R})$ are determined. The formula of the 4-parameter invariant metric on $G_{1}^{J}(\mathbb{R})$ obtained as sum of squares of 6 invariant one-forms is extended to $G_{n}^{J}(\mathbb{R}), n \in \mathbb{N}$. We obtain a three parameter invariant metric on the extended Siegel-Jacobi upper half space $\tilde{X}_{n}^{J} \approx X_{n}^{J} \times \mathbb{R}$ by adding the square of an invariant one-form to the two-parameter balanced metric on the Siegel-Jacobi upper half space $X_{n}^{J}=\frac{G_{n}^{J}(\mathbb{R})}{\mathrm{U}(n) \times \mathbb{R}}$.
\end{abstract}

\section{CONTEnTS}

1. Introduction

2. The Heisenberg group $\mathrm{H}_{n}(\mathbb{R})$ as subgroup of $\operatorname{Sp}(n+1, \mathbb{R})$

3. The symplectic group $\operatorname{Sp}(n, \mathbb{R})$

3.1. Basics

3.2. The real symplectic algebra $\mathfrak{s p}(n, \mathbb{R})$

3.3. $X_{n}$ as Hermitian symmetric space

3.4. Pre-Iwasawa and modified pre-Iwasawa decompositions

4. The real Jacobi group $G_{n}^{J}(\mathbb{R})$

4.1. The Jacobi group $G_{n}^{J}(\mathbb{R})$ as subgroup of $\operatorname{Sp}(n+1, \mathbb{R})$

4.2. The Lie algebra $\mathfrak{g}_{n}^{J}(\mathbb{R})$

4.3. The action

4.4. Fundamental vector fields on $X_{n}^{J}$ and $\tilde{X}_{n}^{J}$

4.5. Invariant one-forms on the Jacobi group

4.6. Invariant vector fields on the Jacobi group

5. Invariant metrics on homogeneous manifolds associated to $G_{n}^{J}(\mathbb{R})$

5.1. Invariant metrics on $X_{n}^{J}$ and $\tilde{X}_{n}^{J}$

6. Appendix: Other representations of the Jacobi algebra

7. Appendix: Differential of square root of a symmetric matrix

2010 Mathematics Subject Classification. 32F45; 53C55; 53B21; 81R30.

Key words and phrases. Jacobi group, Jacobi algebra, Siegel-Jacobi upper half space, extended Siegel-Jacobi upper half space, invariant metrics, coherent states. 


\section{INTRODUCTION}

The real Jacobi group [23, 35, 81, 92] of degree $n$ is defined as $G_{n}^{J}(\mathbb{R}):=\mathrm{H}_{n}(\mathbb{R}) \rtimes$ $\operatorname{Sp}(n, \mathbb{R})$, where $\mathrm{H}_{n}(\mathbb{R})$ denotes the real Heisenberg group. The Siegel-Jacobi upper half space is the $G_{n}^{J}(\mathbb{R})$-homogeneous manifold $X_{n}^{J}:=\frac{G_{n}^{J}(\mathbb{R})}{\mathrm{U}(\mathrm{n}) \times \mathbb{R}} \approx X_{n} \times \mathbb{R}^{2 n}$ [14, 15], [87]-[89], where $\mathcal{X}_{n}$ denotes the Siegel upper half space realized as $\frac{\operatorname{Sp}(n, \mathbb{R})}{\mathrm{U}(\mathrm{n})}$ [44, p 398].

The Jacobi group $G_{n}^{J}:=\mathrm{H}_{n} \rtimes \operatorname{Sp}(n, \mathbb{R})_{\mathbb{C}}$, where $\operatorname{Sp}(n, \mathbb{R})_{\mathbb{C}}:=\operatorname{Sp}(n, \mathbb{C}) \cap \mathrm{U}(n, n)$ [8, 11 ] is also studied in Mathematics, Mathematical Physics and Theoretical Physics, together with the $G_{n}^{J}$-homogeneous Siegel-Jacobi ball $\mathcal{D}_{n}^{J} \approx \mathbb{C}^{n} \times \mathcal{D}_{n}$ [8], where $\mathcal{D}_{n}$ denotes the Siegel ball realized as $\frac{\operatorname{Sp}(n, \mathbb{C})}{\mathrm{U}(n)}$ [44, p 399].

It is well known that $G_{n}^{J}(\mathbb{R}), \operatorname{Sp}(n, \mathbb{R}), \mathrm{H}_{n}(\mathbb{R}), X_{n}^{J}$ and $X_{n}$ are isomorphic with $G_{n}^{J}$, $\operatorname{Sp}(n, \mathbb{R})_{\mathbb{C}}, \mathrm{H}_{n}, \mathcal{D}_{n}^{J}$, respectively $\mathcal{D}_{n}$, see [8]-[12], [16, 23, [35, 87, 88].

The dimensions of the enumerated manifolds are: $\operatorname{dim} \operatorname{Sp}(n, \mathbb{R})=2 n^{2}+n, \operatorname{dim} \mathrm{H}_{n}(\mathbb{R})=$ $2 n+1, \operatorname{dim} G_{n}^{J}(\mathbb{R})=(2 n+1)(n+1), \operatorname{dim} \mathrm{U}(n)=n, \operatorname{dim} X_{n}^{J}=n(n+3), \operatorname{dim} \tilde{X}_{n}^{J}=$ $n(n+3)+1, \operatorname{dim} \mathcal{X}_{n}=n(n+1)$.

The Jacobi group, as a unimodular, non-reductive, algebraic group of Harish-Chandra type [16, 55], [72]-[75], also a coherent state (CS) type group [4, [56, [57, 63, 64, 66] is an interesting object in Mathematics [11, 14. $\mathcal{D}_{n}^{J}$ is a partially bounded domain, nonsymmetric, a Lu Qi-Keng manifold, a projectively induced quantizable Kähler manifold [14], [87, 88].

The Jacobi group has many applications in several branches of Physics: quantum mechanics, geometric quantization, nuclear structure, signal processing, quantum optics, in particular squeezed states and quantum teleportation, see references in [15]. The Jacobi group was known to physicists under other names as Hagen [43], Schrödinger [67], or Weyl-symplectic group [85]. The Jacobi group is responsible for the squeezed states [45, 80, 58, 91, 47] in quantum optics [1, 32, 34, 60, 79].

The Jacobi group was investigated in several publications [7- 11, 14, with Perelomov's CS method [69] based on Kähler manifolds [17]-[20], [56, 57, 63, 64, 66] associated to $G_{n}^{J}$, determining the balanced metric [33]. Berezin's quantization [17]-[20], [26, 27, 36, 71] related to the Jacobi group has been also investigated [14, 24, 25]. But the CS method is applicable only to Kähler manifolds. Because it is desirable to impose the invariance of metric also on manifolds of odd dimension, the CS method must be abandoned.

In this paper we introduce an odd dimensional manifold, called extended Siegel-Jacobi upper half space of order $\mathrm{n}, \tilde{X}_{n}^{J}:=\frac{G_{n}^{J}(\mathbb{R})}{\mathrm{U}(n)} \approx X_{n}^{J} \times \mathbb{R}$, a generalization of the 5 -dimensional Siegel-Jacobi upper half-plane $\tilde{X}_{1}^{J}=\frac{G_{1}^{J}(\mathbb{R})}{\mathrm{SO}(2)} \approx X_{1} \times \mathbb{R}^{3}$ considered in [3, 15]. Because the Jacobi group governs the squeezed states [9, 10], we are expecting that the manifold $\tilde{X}_{n}^{J}$ to have applications in quantum optics. We recall that the squeezed states are a particular class of "minimum uncertainty states" (MUS) [62] and that "Gaussian pure states" ("Gaussons") [78] are more general MUSs.

The invariant metrics on homogeneous manifolds associated to the real Jacobi group $G_{1}^{J}(\mathbb{R})$ were obtained in [3, 15], applying Cartan's moving frame method [28, 29, 37]. We have determined a 3-parameter invariant metric on the extended Siegel-Jacobi upper 
half-plane [3, 15]. To get the invariant metric on $\tilde{X}_{1}^{J}$, we have determined the invariant one-forms $\lambda_{1}, \ldots, \lambda_{6}$ on $G_{1}^{J}(\mathbb{R})$. Then we have calculated the invariant vector fields $L^{j}$ verifying the relations $\left\langle\lambda_{i} \mid L^{j}\right\rangle=\delta_{i j}, i, j=1, \ldots, 6$, such that $L^{j}$ are orthonormal with respect to the 4-parameter invariant metric $\mathrm{d} s_{G_{1}^{J}(\mathbb{R})}^{2}$ expressed in the $S$-coordinates $(x, y, \theta, p, q, \kappa)[23$, p 10], where $\theta \in[0,2 \pi)$ and the other $S$-coordinates are in $\mathbb{R}$.

In the present paper we apply to $G_{n}^{J}(\mathbb{R}), n \in \mathbb{N}$, the method applied in 14 to $G_{1}^{J}(\mathbb{R})$. Firstly we determine the invariant one-forms on $G_{n}^{J}(\mathbb{R})$. If a point $g \in G_{n}^{J}(\mathbb{R})$ is parametrized by the coordinates $(M, X, \kappa)$, where $M \in \operatorname{Sp}(n, \mathbb{R}), X:=(\lambda, \mu) \in$ $M(1,2 n, \mathbb{R}), \kappa \in \mathbb{R}$, and $(p, q)=X M^{-1}$, then we have the following representation of the real Jacobi group embedded in $\operatorname{Sp}(n+1, \mathbb{R})$ [86, 92]

$$
g=\left(\begin{array}{cccc}
a & \mathbb{O}_{n 1} & b & q^{t} \\
\lambda & 1 & \mu & \kappa \\
c & \mathbb{O}_{n 1} & d & -p^{t} \\
\mathbb{O}_{1 n} & 0 & \mathbb{O}_{1 n} & 1
\end{array}\right), M=\left(\begin{array}{cc}
a & b \\
c & d
\end{array}\right) \in \operatorname{Sp}(n, \mathbb{R}) .
$$

In this paper we parametrize the group $G_{n}^{J}(\mathbb{R})$ with a system of coordinates $(x, y, X, Y$, $p, q, \kappa)$, where $x+\mathrm{i} y \in X_{n}, X+\mathrm{i} Y \in \mathrm{U}(n)$, while $(p, q, \kappa)$ characterize the Heisenberg group $\mathrm{H}_{n}(\mathbb{R})$. This system of coordinates, denoted $S_{n}, n \in \mathbb{N}$, coincides for $n=1$ with the $S$-coordinates of $G_{1}^{J}(\mathbb{R})\left[23\right.$, p 10]. The main ingredient of the $S_{n}$-parametrization of $G_{n}^{J}(\mathbb{R})$ is the modified pre-Iwasawa decomposition of the symplectic group $\operatorname{Sp}(n, \mathbb{R})$, inspired by [2, 42]. We obtain a 4-parameter invariant metric on $G_{n}^{J}(\mathbb{R})$, which in the case $n=1$ coincides with the 4-parameter invariant metric determined in [15]. However, the explicit expressions for the metrics in Proposition 2 obtained from the invariant one forms on $G_{n}^{J}(\mathbb{R})$ are quite complicated, so in order to obtain the invariant metric on the odd dimensional extended Siegel-Jacobi space $\tilde{X}_{n}^{J}$ we just add the square of an invariant one-form attached to $\kappa$ to the 2-parameter balanced metric of the Siegel-Jacobi upper half space obtained via the CS method in [11, 14.

The paper is organized as follows. Section 2 summarizes the embedding of the Heisenberg group $\mathrm{H}_{n}(\mathbb{R})$ in $\operatorname{Sp}(n+1, \mathbb{R})$. Section 3 describes the symplectic group. The pre-Iwasawa decomposition is introduced in Lemma 4, while Lemma 5 shows that the modified pre-Iwasawa decomposition is compatible with the linear fractional action of $\operatorname{Sp}(n, \mathbb{R})$ on $X_{n}$. Section 4 considers the real Jacobi group $G_{n}^{J}(\mathbb{R})$. The embedding of $G_{n}^{J}(\mathbb{R})$ in $\operatorname{Sp}(n+1, \mathbb{R})$ is described in Remark 6 . After choosing a base of the Lie algebra $\mathfrak{g}_{n}^{J}(\mathbb{R})$ which in particular for $n=1$ coincides with that in [15], Lemma 8 describes the action of the Jacobi group on the homogeneous manifolds $\mathcal{X}_{n}^{J}$ and $\tilde{X}_{n}^{J}$. In Section 4.4 are calculated the fundamental vector fields (FVF) associated to the generators of the Jacobi group on $X_{n}^{J}$ and $\tilde{X}_{n}^{J}$. In Section 4.5 are obtained the invariant one-forms on $G_{n}^{J}(\mathbb{R})$ in the $S_{n}$-coordinates, see Lemma 10 and (4.32). The difficulties to calculate the invariant vector fields once the invariant one-forms are known are exemplified in Section 4.6. Proposition 2 expresses the 4-parameter invariant metric on $G_{n}^{J}(\mathbb{R})$. Proposition 3. an extension to $n \in \mathbb{N}$ of [3. Proposition1], expresses the Kähler two-form on $X_{n}^{J}$ in several types of variables. Remark 11 gives a CS-meaning to the $S_{n}$-parameters $p, q$ describing $G_{n}^{J}(\mathbb{R})$. The invariant metric on the odd dimensional manifold $\tilde{X}_{n}^{J}$ is given in Theorem 11. Finally, other parametrizations of the Jacobi algebra $\mathfrak{g}_{n}^{J}(\mathbb{R})$ are recalled 
in $\S$ 6, while Section 7 summarizes the method of calculating the differential of square root of a symmetric matrix.

To conclude, the new results of this paper are contained in Lemma 4, Lemma 5, parts of Lemma 8, the base (4.6) of $\mathfrak{g}_{n}^{J}(\mathbb{R})$, Lemma 10, Propositions 1 - 3 and Remark 11, The main result of the present investigation is stated in Theorem 1,

Notation We denote by $\mathbb{R}, \mathbb{C}, \mathbb{Z}$, and $\mathbb{N}$ the field of real numbers, the field of complex numbers, the ring of integers, and the set of positive integers, respectively. We denote by i the imaginary unit $\sqrt{-1}$, and the complex conjugate of $z$ by $\bar{z}$. We denote the set of $m \times n$ matrice with entries in the field $\mathbb{F}$ as $M(m, n ; \mathbb{F})$ and if $n=m$ we write $M(n, \mathbb{F})$. $M(n, \mathbb{F})$ for $\mathbb{E}$ equal with $\mathbb{R}$ or $\mathbb{C}$ is denoted by $M(n)$. We denote the transpose (the Hermitian conjugate) of the matrix $A$ by $A^{t}$, (respectively $A^{\dagger}$ ). $\mathbb{1}_{n}$ denotes the identity matrix of $M(n, \mathbb{F})$, while $\mathbb{O}_{n m} \in M(n, m, \mathbb{F})$ denotes the matrix with all elements zero and $\mathbb{O}_{n}$ means $\mathbb{O}_{n n}$. $E_{p} \in M(1, n, \mathbb{R})$ denotes the matrix with 1 on the position $p$, $\left(E_{p}\right)_{i}=\delta_{p i}$ and similarly for $E_{q}, p, q=1, \ldots, n$. $E_{i j}$ denotes the square matrix with entry 1 at the intersection of the $i$ th row with the $j$ th column, $\left(E_{i j}\right)_{k l}=\delta_{i k} \delta_{j l}$, and $E_{i j} E_{k l}=\delta_{j k} E_{i l}$. When the dimension of a submatrix of a block matrix is not evident, the subindices $p q$ specify that the respective submatrix is in $M(p, q, \mathbb{R})$. We denote by $\mathrm{d}$ the differential. We use Einstein convention that repeated indices are implicitly summed over. We denote by $\operatorname{dg}\left(a_{1}, \ldots, a_{n}\right)$ the diagonal matrix which has on diagonal $a_{1}, \ldots, a_{n}$. We denote by $\langle\lambda \mid L\rangle$ the pairing of the one-form $\lambda$ with the vector field $L$. We consider a complex separable Hilbert space $\mathfrak{H}$ endowed with a scalar product $(\cdot, \cdot)$ which is antilinear in the first argument, $(\lambda x, y)=\bar{\lambda}(x, y) x, y \in \mathfrak{H}, \lambda \in \mathbb{C} \backslash 0$. If $\pi$ is a representation of a Lie group $G$ on the Hilbert space $\mathfrak{H}$ and $\mathfrak{g}$ is the Lie algebra of $G$, we denote $\boldsymbol{X}:=\mathrm{d} \pi(X)$ for $X \in \mathfrak{g}$.

\section{The Heisenberg group $\mathrm{H}_{n}(\mathbb{R})$ as subgroup of $\operatorname{Sp}(n+1, \mathbb{R})$}

The real Heisenberg group $\mathrm{H}_{n}(\mathbb{R})$, parametrized by $(\lambda, \mu, \kappa), \lambda, \mu \in M(1, n, \mathbb{R}), \kappa \in \mathbb{R}$, has the composition law [16, 55, 74, 75, 86, ,92]

$$
(\lambda, \mu, \kappa) \times\left(\lambda^{\prime}, \mu^{\prime}, \kappa^{\prime}\right)=\left(\lambda+\lambda^{\prime}, \mu+\mu^{\prime}, \kappa+\kappa^{\prime}+\lambda \mu^{\prime t}-\mu \lambda^{\prime t}\right)
$$

$\mathrm{H}_{n}(\mathbb{R})$ is a particular case of the Heisenberg group $H_{\mathbb{R}}^{(n, m)}$ for $m=1$, see [87] and [90].

If $g \in \mathrm{H}_{n}(\mathbb{R})$, we represent it [86, 92 and its inverse embedded in $\operatorname{Sp}(n+1, \mathbb{R})$ as

$$
g=\left(\begin{array}{cccc}
1 & 0 & 0 & \mu^{t} \\
\lambda & 1 & \mu & \kappa \\
0 & 0 & 1 & -\lambda^{t} \\
0 & 0 & 0 & 1
\end{array}\right), g^{-1}=\left(\begin{array}{cccc}
1 & 0 & 0 & -\mu^{t} \\
-\lambda & 1- & \mu & -\kappa \\
0 & 0 & 1 & \lambda^{t} \\
0 & 0 & 0 & 1
\end{array}\right)
$$

see also notation in (1.1) and Lemma 6. 
If the generators $P_{p}, Q_{q}, p, q=1, \ldots, n, R$, of the Heisenberg group are defined in (2.3), see also the last three equations in (4.5) and Lemma 6 ,

$$
\begin{aligned}
P_{p} & =\left(\begin{array}{cccc}
0 & 0 & 0 & 0 \\
E_{p} & 0 & 0 & 0 \\
0 & 0 & 0 & -E_{p}^{t} \\
0 & 0 & 0 & 0
\end{array}\right), p=1, \ldots, n \\
Q_{q} & =\left(\begin{array}{cccc}
0 & 0 & 0 & E_{q}^{t} \\
0 & 0 & E_{q} & 0 \\
0 & 0 & 0 & 0 \\
0 & 0 & 0 & 0
\end{array}\right), q=1, \ldots, n, \\
R & =\left(\begin{array}{cccc}
0 & 0 & 0 & 0 \\
0 & 0 & 0 & 1 \\
0 & 0 & 0 & 0 \\
0 & 0 & 0 & 0
\end{array}\right)
\end{aligned}
$$

then

$$
g^{-1} \mathrm{~d} g=P_{p} \lambda^{p}+Q_{q} \lambda^{q}+R \lambda^{r} .
$$

With (2.2) and (2.4), the left invariant one-forms on $\mathrm{H}_{n}(\mathbb{R})$ are

$$
\lambda^{p}=\mathrm{d} \lambda_{p}, \quad \lambda^{q}=\mathrm{d} \mu_{q}, \quad \lambda^{r}=\mathrm{d} \kappa-\lambda \mathrm{d} \mu^{t}+\mu \mathrm{d} \lambda^{t} .
$$

The left action of the Heisenberg group on itself is obtained from (2.1)

$$
\exp \left(\lambda P+\mu^{t} Q+\kappa R\right)\left(\lambda_{0}, \mu_{0}, \kappa_{0}\right)=\left(\lambda+\lambda_{0}, \mu+\mu_{0}, \kappa+\kappa_{0}+\lambda \mu_{0}^{t}-\mu \lambda_{0}^{t}\right) .
$$

The left invariant metric on the Heisenberg group is

$$
g^{L}(\lambda, \mu, \kappa)=\mathrm{d} \lambda^{2}+\mathrm{d} \mu^{2}+\left(\mathrm{d} \kappa-\lambda \mathrm{d} \mu^{t}+\mu \mathrm{d} \lambda^{t}\right)^{2}
$$

The fundamental vector fields, see [44, p. 121, Ch II $\S 3]$, [53, p. 42], or [15, $\S 6.1$, v1], on the Heisenberg group $\mathrm{H}_{n}(\mathbb{R})$ are

$$
P^{*}=\frac{\partial}{\partial_{\lambda}}+\mu^{t} \frac{\partial}{\partial_{\kappa}}, \quad Q^{*}=\frac{\partial}{\partial_{\mu}}-\lambda \frac{\partial}{\partial_{\kappa}}, \quad R^{*}=\frac{\partial}{\partial_{\kappa}} .
$$

See also (4.25).

\section{The SymplectiC Group $\operatorname{Sp}(n, \mathbb{R})$}

3.1. Basics. The group $\operatorname{Sp}(n, \mathbb{K})$ admits a matrix realization in $M \in \mathrm{M}(2 n, \mathbb{K})$, where $\mathbb{K}$ is $\mathbb{R}$ or $\mathbb{C}$, verifying the relation

$$
M^{t} J_{n} M=J_{n}, \quad J_{n}:=\left(\begin{array}{cc}
\mathbb{O}_{n} & \mathbb{1}_{n} \\
-\mathbb{1}_{n} & \mathbb{O}_{n}
\end{array}\right) .
$$

If there is no possibility of confusion, we denote $J_{n}$ just with $J$.

Let us consider a matrix

$$
M=\left(\begin{array}{ll}
a & b \\
c & d
\end{array}\right), \quad a, b, c, d \in M(n, \mathbb{R}) .
$$

It is easy to prove [38]-[40], [77] that 
Remark 1. a) If $M \in \operatorname{Sp}(n, \mathbb{R})$, then $M$ is similar with $M^{t}$ and $M^{-1}$ and also $J \in$ $\operatorname{Sp}(n, \mathbb{R})$.

b) If $M \in \operatorname{Sp}(n, \mathbb{R})$ is as in (3.2), then the matrices $a, b, c, d$ in (3.2) verify the sets of equivalent conditions

$$
\begin{aligned}
& a b^{t}-b a^{t}=\mathbb{O}_{n}, a d^{t}-b c^{t}=\mathbb{1}_{n}, c d^{t}-d c^{t}=\mathbb{O}_{n} \\
& a^{t} c-c^{t} a=\mathbb{O}_{n}, \quad a^{t} d-c^{t} b=\mathbb{1}_{n}, \quad b^{t} d-d^{t} b=\mathbb{O}_{n} .
\end{aligned}
$$

c) If $M \in \operatorname{Sp}(n, \mathbb{R})$ has the form (3.2), then

$$
M^{-1}=\left(\begin{array}{cc}
d^{t} & -b^{t} \\
-c^{t} & a^{t}
\end{array}\right)
$$

d) The matrices in $\operatorname{Sp}(n, \mathbb{R})$ have the determinant 1 .

e) The following subsets of $\operatorname{GL}(2 n, \mathbb{R})$ are subgroups of $\operatorname{Sp}(n, \mathbb{R})$

$$
\begin{gathered}
N=\left\{\left(\begin{array}{ll}
\mathbb{1}_{n} & A \\
\mathbb{O}_{n} & \mathbb{1}_{n}
\end{array}\right): A=A^{t}\right\}, \tilde{N}=\left\{\left(\begin{array}{cc}
\mathbb{1}_{n} & \mathbb{O}_{n} \\
B & \mathbb{1}_{n}
\end{array}\right): B=B^{t}\right\}, \\
D=\left\{\left(\begin{array}{cc}
C & \mathbb{O}_{n} \\
\mathbb{O}_{n} & \left(C^{t}\right)^{-1}
\end{array}\right): C \in \mathrm{GL}(n, \mathbb{R})\right\} .
\end{gathered}
$$

$\operatorname{Sp}(n, \mathbb{R})$ is generated by $D \cup \tilde{N} \cup\{J\}$ and $D \cup N \cup\{J\}$.

Using (3.4) it can be shown that the matrix $\mathcal{M} \in \mathrm{Sp}(n, \mathbb{R}) \cap \mathrm{O}_{2 n}$ has the expression

$$
\mathcal{M}=\left(\begin{array}{cc}
X & Y \\
-Y & X
\end{array}\right), X^{t} X+Y^{t} Y=X X^{t}+Y Y^{t}=\mathbb{1}_{n}, X^{t} Y=Y^{t} X, Y X^{t}=X Y^{t}
$$

If $\mathcal{M} \in M(2 n, \mathbb{R})$ has the properties (3.5), let

$$
\mathcal{M}^{\prime}:=X+\mathrm{i} Y \in M(n, \mathbb{C}),
$$

and

Remark 2. The correspondence $\mathcal{M} \rightarrow \mathcal{M}^{\prime}$ of $(3.5)$ with (3.6) is a group isomorphism

$$
\operatorname{Sp}(n, \mathbb{R}) \cap \mathrm{O}_{2 n} \approx \mathrm{U}(n) .
$$

3.2. The real symplectic algebra $\mathfrak{s p}(n, \mathbb{R})$. The real symplectic Lie algebra $\mathfrak{g}=$ $\mathfrak{s p}(n, \mathbb{R})$ is a real form of the simple Lie algebra $\mathfrak{s p}(n, \mathbb{C})$ of type $\mathfrak{c}_{n}$ and $X \in \mathfrak{s p}(n, \mathbb{R})$ if $X^{t} J+J X=0$, or equivalently

$$
X=\left(\begin{array}{cc}
a & b \\
c & -a^{t}
\end{array}\right), \quad b=b^{t}, \quad c=c^{t},
$$

where $a, b, c \in M(n, \mathbb{R})$, and similarly for $\mathfrak{s p}(n, \mathbb{C})$.

We write an element $X$ (3.7) as

$$
X=\sum_{i, j} a_{i j} H_{i j}+2 \sum_{i<j}\left(b_{i j} F_{i j}+c_{i j} G_{i j}\right)+\sum_{i=j}\left(b_{i j} F_{i j}+c_{i j} G_{i j}\right), \quad 1 \leq i, j \leq n,
$$

$$
H_{i j}:=\left(\begin{array}{cc}
E_{i j} & \mathbb{O}_{n} \\
\mathbb{O}_{n} & -E_{j i}
\end{array}\right), 2 F_{i j}:=\left(\begin{array}{cc}
\mathbb{O}_{n} & E_{i j}+E_{j i} \\
\mathbb{O}_{n} & \mathbb{O}_{n}
\end{array}\right) ; 2 G_{i j}:=\left(\begin{array}{cc}
\mathbb{O}_{n} & \mathbb{O}_{n} \\
E_{i j}+E_{j i} & \mathbb{O}_{n}
\end{array}\right) .
$$


In the matrix realization (3.7), the real algebra $\mathfrak{s p}(n, \mathbb{R})$ has the $2 n^{2}+n$ generators

$$
H_{i j}, \quad F_{i j}, \quad G_{i j}, \quad 1 \leq i \leq j \leq n .
$$

3.3. $X_{n}$ as Hermitian symmetric space. We briefly recall some well known facts about Hermitian symmetric spaces [8, 44, 84]. We use the notation

$X_{n}$ : Hermitian symmetric space of noncompact type, $X_{n}=G_{0} / K$;

$X_{c}$ : compact dual of $X_{n}, X_{c}=G_{c} / K$;

$G_{0}$ : real Hermitian group;

$G=G_{0}^{\mathbb{C}}$ : the complexification of $G_{0}$;

$P: \quad$ a parabolic subgroup of $G$;

$K$ : maximal compact subgroup of $G_{0}$;

$G_{c}$ : compact real form of $G$.

The compact manifold $X_{c}$ of $\frac{n(n+1)}{2}$-complex dimension has a complex structure inherited from the identification of $X_{c}$ with $G / P$. The group $G_{c}$ acts transitively on $X_{c}$ with isotropy group $K=G_{0} \cap P=G_{c} \cap P$.

$X_{n}=G_{0} / K=G_{n}\left(x_{0}\right)$ is open in $X_{c}$, where $x_{0}$ is a base point of $G$ corresponding to $K$. If $\left\{e_{1}, \ldots, e_{2 n}\right\}$ is a base of $\mathbb{C}^{2 n}$, in our case we take $x_{0}=e_{n+1} \wedge \cdots \wedge e_{2 n} \in X_{n}$ as base point, and $G_{0}=\operatorname{Sp}(n, \mathbb{R}) \approx \operatorname{Sp}(n, \mathbb{R})_{\mathbb{C}}$.

$X_{c}$ includes $X_{n}$ under Borel embedding $X_{n} \subset X_{c}: g K \mapsto g P, g \in G_{0}$.

The hermitian form on $\mathbb{C}^{2 n}$

$$
<u, v>=-\sum_{j=1}^{n} u^{j} \bar{v}^{j}+\sum_{k=1}^{n} u^{n+k} \bar{v}^{n+k}
$$

specifies the indefinite unitary group $\mathrm{U}(n, n)$, hence the transformation group $\operatorname{Sp}(n, \mathbb{R})_{\mathbb{C}}$ acting on $X_{n}$

$$
G:=\operatorname{Sp}(n, \mathbb{C}), G_{c}:=\operatorname{Sp}(n)=\operatorname{Sp}(n, \mathbb{C}) \cap \mathrm{U}(2 n) \subset \mathrm{SU}(2 n), K:=\mathrm{U}(n) .
$$

We have also

$$
P:=\left\{g \in G: g\left(x_{0}\right)=x_{0}\right\}=\left\{\left(\begin{array}{cc}
a & \mathbb{O}_{n} \\
c & d
\end{array}\right): a^{t} c=c^{t} a, a^{t} d=\mathbb{1}_{n}\right\} .
$$

Let us consider also

$$
\mathfrak{m}^{+}:=\left\{\left(\begin{array}{cc}
\mathbb{O}_{n} & b \\
\mathbb{O}_{n} & \mathbb{O}_{n}
\end{array}\right): b^{t}=b\right\}, b \in M(n, \mathbb{C})
$$

Then

$$
W \mapsto \hat{W}=\left(\begin{array}{cc}
\mathbb{O}_{n} & W \\
\mathbb{O}_{n} & \mathbb{O}_{n}
\end{array}\right), \xi(W)=(\exp \hat{W}) x_{0}=v_{1} \wedge \cdots \wedge v_{n},\left(v_{1}, \cdots, v_{n}\right)=\left(\begin{array}{l}
W \\
\mathbb{1}_{n}
\end{array}\right)
$$

and $\xi$ maps the symmetric $n \times n$ matrices $W$ of $\mathfrak{m}^{+}$such that $\mathbb{1}_{n}-W \bar{W}>0$ onto a dense open subset of $X_{c}$ that contains $X_{n}$.

$X_{n}$ is a Hermitian symmetric space of type CI (cf. Table V, p. 518, in [44]), identified with the symmetric bounded domain of type II, $\mathfrak{R}_{I I}$ in Hua's notation [51].

Let us denote by $X_{n}$ the set

$$
X_{n}:=\left\{v \in M(n, \mathbb{C}) \mid v=s+\mathrm{i} r, s, r \in M(n, \mathbb{R}), r>0, s^{t}=s ; r^{t}=r\right\} .
$$


Remark 3. The action (3.12) of $\operatorname{Sp}(n, \mathbb{R})$ on the Siegel upper half space $X_{n}$

$$
v_{1}=M(v)=(a v+b)(c v+d)^{-1}=\left(v c^{t}+d^{t}\right)^{-1}\left(v a^{t}+b^{t}\right) .
$$

is a transitive one. The correspondence

$$
\zeta: X_{n} \rightarrow X_{n}=\operatorname{Sp}(n, \mathbb{R}) / K, K=\operatorname{Sp}(n, \mathbb{R}) \cap \mathrm{O}_{2 n} ; v \mapsto M_{X+\mathrm{i} Y} K,
$$

where $M_{X+\mathrm{i} Y}$ is defined in (3.13), is a 1-1 map which realizes the Siegel upper half space (3.11) as the homogenous manifold $X_{n}$.

Proof. Firstly it is proved that the matrix $c v+d$ in (3.12) is invertible, see e.g. [39, pp 1-11]. Then it is proved that $M(v) \in X_{n}$ [39, 177].

It is find a symplectic map that sends i $\mathbb{1}_{n}$ to $X+\mathrm{i} Y \in \mathcal{X}_{n}, Y>0$ as the composition of the symplectic maps $V \rightarrow \sqrt{Y} V \sqrt{Y}$ and $V \rightarrow V+X$ associated with the symplectic matrices

$$
\left(\begin{array}{cc}
\sqrt{Y} & \mathbb{O}_{n} \\
\mathbb{O}_{n} & \sqrt{Y^{-1}}
\end{array}\right) \text { and }\left(\begin{array}{cc}
\mathbb{1}_{n} & X \\
\mathbb{O}_{n} & \mathbb{1}_{n}
\end{array}\right) .
$$

We introduce the notation

$$
M_{X+\mathrm{i} Y}:=\left(\begin{array}{ll}
\mathbb{1}_{n} & X \\
\mathbb{0}_{n} & \mathbb{1}_{n}
\end{array}\right)\left(\begin{array}{cc}
\sqrt{Y} & \mathbb{0}_{n} \\
\mathbb{O}_{n} & \sqrt{Y^{-1}}
\end{array}\right)=\left(\begin{array}{cc}
\sqrt{Y} & X \sqrt{Y^{-1}} \\
\mathbb{O}_{n} & \sqrt{Y^{-1}}
\end{array}\right) .
$$

The subgroup of $\operatorname{Sp}(n, \mathbb{R})$ which stabilizes $\mathrm{i} \mathbb{1}_{n} \in X_{n}$ is the subgroup of orthogonal symplectic matrices of the form (3.5).

Note that an argument similar with that used in Remark 3 was given in [1], following 78.

3.4. Pre-Iwasawa and modified pre-Iwasawa decompositions. We recall that the Iwasawa decomposition [44, Ch VI, §3] of $\mathrm{SL}(2, \mathbb{R})$ is used for the so called $S$ parametrization of the Jacobi group $G_{1}^{J}(\mathbb{R})$, see [21, p 4], [22, p 15], [23, p 7].

In the present paper we find a similar decomposition for $G_{n}^{J}(\mathbb{R})$.

We recall the Iwasawa decomposition [44, Ch. VI, §3] of $\operatorname{Sp}(n, \mathbb{R}) \ni G=K A N$ [83, p. 285] corresponds to

$$
\begin{gathered}
K:=\left\{\left(\begin{array}{cc}
A & B \\
-B & A
\end{array}\right) \in \mathrm{U}(n)\right\}, \\
A:=\left\{\operatorname{diag}\left(a_{1}, \ldots, a_{n}, a_{1}^{-1}, \ldots, a_{n}^{-1}\right) ; a_{1}, \ldots, a_{n}>0\right\}, \\
N:=\left\{\left(\begin{array}{cc}
A & B \\
\mathbb{O}_{n} & \left(A^{-1}\right)^{t}
\end{array}\right): \text { A real unit upper triangular, } A B^{t}=B A^{t}\right\} .
\end{gathered}
$$

For Cholesky factorisation see [83, p. 287] and [82]; for QR decomposition see [76, p. 143]. We also mention the Iwasawa decomposition for $G_{n}^{J}(\mathbb{R})$ was considered in [87, $\S 9.1 .2]$.

Following the method of [2] and [42, §2.2.2], we find similarly

Lemma 4. Pre-Iwasawa decomposition Let as consider the pre-Iwasawa decomposition of $M \in \operatorname{Sp}(n, \mathbb{R})$

$$
M=\left(\begin{array}{ll}
a & b \\
c & d
\end{array}\right)=\left(\begin{array}{cc}
\mathbb{1}_{n} & x \\
\mathbb{O}_{n} & \mathbb{1}_{n}
\end{array}\right)\left(\begin{array}{cc}
y & \mathbb{O}_{n} \\
\mathbb{O}_{n} & y^{-1}
\end{array}\right)\left(\begin{array}{cc}
X & Y \\
-Y & X
\end{array}\right),
$$


where the last matrix in (3.14) in $\mathrm{U}(n)$ verify (3.5).

We find

$$
y=\left(d d^{t}+c c^{t}\right)^{-\frac{1}{2}}, X-\mathrm{i} Y=y(d+\mathrm{i} c) .
$$

Let us also define

$$
t:=y^{2}\left(d b^{t}+c a^{t}\right) y^{-1}=\left(b d^{t}+a c^{t}\right) y .
$$

The matrices $y$ and $x:=$ ty are symmetric, $y$ is positive definite, and all the factors in (3.14) are unique. We have also

$$
x=\left(d d^{t}+c c^{t}\right)^{-1}\left(d b^{t}+c a^{t}\right)=\left(b d^{t}+a c^{t}\right)\left(d d^{t}+c c^{t}\right)^{-1} .
$$

The inverse of the transform $(a, b, c, d) \rightarrow(x, y, X, Y)$ in equations (3.15), (3.16) is

$$
a=y X-x y^{-1} Y, b=y Y+x y^{-1} X, c=-y^{-1} Y, d=y^{-1} X .
$$

In the case of $\mathrm{SL}(2, \mathbb{R})$, the expresion (3.17) corresponds to (46.b) in [15] if we replace $y \rightarrow y^{1 / 2}$ and take $X=\cos \theta, Y=\sin \theta$.

The first factor in (3.14) corresponds to the "free propagation subgroup" [2].

In the next Lemma we modify the pre-Iwasawa decomposition of $\operatorname{Sp}(n, \mathbb{R})$ so that it coincides with Iwasawa decomposition of the group $\operatorname{Sp}(1, \mathbb{R}) \approx \operatorname{SL}(2, \mathbb{R})$ in $[23$, p 9].

We get

Lemma 5. Modified pre-Iwasawa decomposition The action of $M \in \operatorname{Sp}(n, \mathbb{R})$ (3.2) on $X_{n}$, expressed in the parameters of the pre-Iwasawa decomposition in Lemma 4

$$
(a, b, c, d) \times\left(x^{\prime}, y^{\prime}, X^{\prime}, Y^{\prime}\right) \rightarrow\left(x_{1}, y_{1}, X_{1}, Y_{1}\right),
$$

where $x^{\prime}, y^{\prime} \in M(n, \mathbb{R}), x^{\prime}=\left(x^{\prime}\right)^{t}, y^{\prime}=\left(y^{\prime}\right)^{t}, y^{\prime}>0$, and

$$
\begin{aligned}
& a=y^{1 / 2} X-x y^{-1 / 2} Y, b=y^{1 / 2} Y+x y^{-1 / 2} X, c=-y^{-1 / 2} Y, d=y^{-1 / 2} X, \\
& x=y\left(d b^{t}+c a^{t}\right), y=\left(d d^{t}+c c^{t}\right)^{-1}, X-\mathrm{i} Y=y^{\frac{1}{2}}(d+\mathrm{i} c),
\end{aligned}
$$

is given by the formulas

$$
\begin{gathered}
x_{1}+\mathrm{i} y_{1}=\left[c\left(y^{\prime}+x^{\prime} y^{\prime-1} x^{\prime}\right) c^{t}+d\left(y^{\prime}\right)^{-1} d^{t}+c x^{\prime}\left(y^{\prime}\right)^{-1} d^{t}+d\left(y^{\prime}\right)^{-1} x^{\prime} c^{t}\right]^{-1} \\
\times\left[c\left(y^{\prime}+x^{\prime}\left(y^{\prime}\right)^{-1} x^{\prime}\right) a^{t}+c x^{\prime}\left(y^{\prime}\right)^{-1} b^{t}+d\left(y^{\prime}\right)^{-1} x^{\prime} a^{t}+d\left(y^{\prime}\right)^{-1} b^{t}+\mathrm{i}\right], \\
X_{1}-\mathrm{i} Y_{1}=\left(y_{1}\right)^{1 / 2}\left\{\left(c x^{\prime}+d\right)\left(y^{\prime}\right)^{-1 / 2} X^{\prime}+c\left(y^{\prime}\right)^{1 / 2} Y^{\prime}\right. \\
\left.\quad+\mathrm{i}\left[c\left(y^{\prime}\right)^{1 / 2} X^{\prime}-\left(c x^{\prime}+d\right)\left(y^{\prime}\right)^{-1 / 2} Y^{\prime}\right]\right\}
\end{gathered}
$$

while the action given by (3.12) $M \times\left(x^{\prime}, y^{\prime}\right) \rightarrow\left(\mathrm{x}_{1}, \mathrm{y}_{1}\right), \mathrm{v}_{1}:=\mathrm{x}_{1}+\mathrm{i} \mathrm{y}_{1}$ expressing the linear fractional (3.12) transformation is

$$
\begin{aligned}
\mathrm{x}_{1}+\mathrm{i}_{1} & =\left(\bar{v}^{\prime} c^{t}+d^{t}\right)^{-1}\left(\frac{B}{2}+\mathrm{i} y^{\prime}\right)\left(c v^{\prime}+d\right)^{-1}, \\
B & =2 \bar{v}^{\prime} a^{t} c v^{\prime}+\bar{v}^{\prime}\left(c^{t} b+a^{t} d\right)+\left(b^{t} c+d^{t} a\right) v^{\prime}+2 b^{t} d .
\end{aligned}
$$

The modified pre-Iwasawa decomposition (3.19) is compatible with the Möbius transform (3.22), i. e.

$$
x_{1}+\mathrm{i} y_{1} \equiv \mathrm{x}_{1}+\mathrm{i} \mathrm{y}_{1}
$$


The transformation of the matrices associated as in Remark 2 to the pair $(X, Y)$ defined in (3.21) under the action (3.18) reads

$$
\left(\begin{array}{cc}
X_{1} & Y_{1} \\
-Y_{1} & X_{1}
\end{array}\right)=y_{1}^{\frac{1}{2}}\left[\left(c x^{\prime}+d\right)\left(y^{\prime}\right)^{-\frac{1}{2}} \mathbb{1}_{2 n}-c\left(y^{\prime}\right)^{\frac{1}{2}} J_{2 n}\right]\left(\begin{array}{cc}
X^{\prime} & Y^{\prime} \\
-Y^{\prime} & X^{\prime}
\end{array}\right) .
$$

Proof. This is an easy but long calculation and we indicate only the main steps.

We write $(3.20)$ as

$$
x_{1}+\mathrm{i} y_{1}=A^{-1} M
$$

where

$$
\begin{aligned}
A & :=c\left(y^{\prime}+x^{\prime} y^{\prime-1} x^{\prime}\right) c^{t}+d\left(y^{\prime}\right)^{-1} d^{t}+c x^{\prime}\left(y^{\prime}\right)^{-1} d^{t}+d\left(y^{\prime}\right)^{-1} x^{\prime} c^{t}, \\
M & :=c\left(y^{\prime}+x^{\prime}\left(y^{\prime}\right)^{-1} x^{\prime}\right) a^{t}+c x^{\prime}\left(y^{\prime}\right)^{-1} b^{t}+d\left(y^{\prime}\right)^{-1} x^{\prime} a^{t}+d\left(y^{\prime}\right)^{-1} b^{t} .
\end{aligned}
$$

Firstly it is proved that $y_{1}$ defined in (3.20) is equal with $\mathrm{y}_{1}$ in (3.22) and then it is obtained

$$
A=\left(c v^{\prime}+d\right) y^{\prime-1}\left(\bar{v}^{\prime} c^{t}+d^{t}\right)
$$

or

$$
A^{-1}=\left(\bar{v}^{\prime} c^{t}+d^{t}\right)^{-1} y^{\prime}\left(c v^{\prime}+d\right)^{-1} .
$$

In order to prove that $x_{1}$ in (3.20) is equal with $\mathrm{x}_{1}$ in (3.22), with (3.24), we have to verify that

i.e.

$$
y^{\prime}\left(c v^{\prime}+d\right)^{-1} M=\frac{B}{2}\left(c v^{\prime}+d\right)^{-1},
$$

$$
M\left(c v^{\prime}+d\right)=\left(c v^{\prime}+d\right)\left(y^{\prime}\right)^{-1} \frac{B}{2} .
$$

Using equations (3.3), it is verified the identity of the imaginary parts of both sides of (3.25) and then the identity of the real parts.

\section{The REAL Jacobi Group $G_{n}^{J}(\mathbb{R})$}

The real Jacobi group $G_{n}^{J}(\mathbb{R})$ has the composition law

$$
(M,(\lambda, \mu, \kappa)) \times\left(M^{\prime},\left(\lambda^{\prime}, \mu^{\prime}, \kappa^{\prime}\right)\right)=\left(M M^{\prime},\left(\tilde{\lambda}+\lambda^{\prime}, \tilde{\mu}+\mu^{\prime}, \kappa+\kappa^{\prime}+\tilde{\lambda} \mu^{\prime t}-\tilde{\mu} \lambda^{\prime t}\right),\right.
$$

where $M, M^{\prime} \in \operatorname{Sp}(n, \mathbb{R})$ have the form (3.2) and verifies the conditions of 3.3 , $(\lambda, \mu, \kappa)$, $\left(\lambda^{\prime}, \mu^{\prime}, \kappa^{\prime}\right) \in \mathrm{H}_{n}(\mathbb{R})$, and $(\tilde{\lambda}, \tilde{\mu})=(\lambda, \mu) M^{\prime}$ [16, 55, 74, 75, 87, 92].

4.1. The Jacobi group $G_{n}^{J}(\mathbb{R})$ as subgroup of $\mathbf{S p}(n+1, \mathbb{R})$. Let us consider a matrix $M \in \operatorname{Sp}(n, \mathbb{R})$ as in $(3.2)$ verifying (3.3). Let us introduce the block matrix

$$
g:=\left(\begin{array}{cc}
A & B \\
C & D
\end{array}\right) \in M(2 n+2, \mathbb{R})
$$

where the submatrices $A, B, C, D \in M(n+1, \mathbb{R})$ are defined as in (1.1), i.e.

$$
A:=\left(\begin{array}{ll}
a_{n n} & \mathbb{O}_{n 1} \\
\lambda_{1 n} & 1_{11}
\end{array}\right), B:=\left(\begin{array}{ll}
b_{n n} & q_{n 1}^{t} \\
\mu_{1 n} & \kappa_{11}
\end{array}\right), C:=\left(\begin{array}{cc}
c_{n n} & \mathbb{O}_{n 1} \\
\mathbb{O}_{1 n} & 0
\end{array}\right), D:=\left(\begin{array}{cc}
d_{n n} & -p_{n 1}^{t} \\
\mathbb{O}_{1 n} & 1_{11}
\end{array}\right) .
$$

We verify that indeed 
Lemma 6. The matrix $g$ defined in (4.2), (4.3) is in $\operatorname{Sp}(n+1, \mathbb{R})$.

Proof. We calculate the submatrices of the matrix $L$

$$
L:=g J_{n+1} g^{t}=\left(\begin{array}{cc}
U & V \\
Z & T
\end{array}\right) .
$$

We find

$$
U=\mathbb{O}_{n+1}, V=\mathbb{1}_{n+1}, Z=-\mathbb{1}_{n+1}, T=\mathbb{O}_{n+1},
$$

i.e. $L=J_{n+1}$ and the conditions (3.1) are verified.

If $g=(M, X, \kappa) \in G_{n}^{J}(\mathbb{R})$, then $g^{-1}=\left(M^{-1},-Y,-\kappa\right)$, i.e., with the conventions in (4.2), (4.3), we have the following representation in $\operatorname{Sp}(n+1, \mathbb{R})$, see also (1.1)

$$
g=\left(\begin{array}{cccc}
a & 0 & b & q^{t} \\
\lambda & 1 & \mu & \kappa \\
c & 0 & d & -p^{t} \\
0 & 0 & 0 & 1
\end{array}\right), g^{-1}=\left(\begin{array}{cccc}
d^{t} & 0 & -b^{t} & -\mu^{t} \\
-p & 1 & -q & -\kappa \\
-c^{t} & 0 & a^{t} & \lambda^{t} \\
0 & 0 & 0 & 1
\end{array}\right), M=\left(\begin{array}{ll}
a & b \\
c & d
\end{array}\right) \in \operatorname{Sp}(n, \mathbb{R})
$$

4.2. The Lie algebra $\mathfrak{g}_{n}^{J}(\mathbb{R})$. Now we introduce a set of matrices that form a base for the Lie algebra $\mathfrak{g}_{n}^{J}(\mathbb{R})$ embedded in $\mathfrak{s p}(n+1, \mathbb{R})$ as in Lemma 6 which in the case $n=1$ corresponds to the base $F, G, H, P, Q, R$ in [15]

$$
\begin{aligned}
2\left(F_{I J}\right)_{i j} & :=\delta_{I, i} \delta_{J, n+1+j}+\delta_{I, j} \delta_{J, n+1+i}, I, J=1, \ldots, 2 n+2 ; i, j=1, \ldots, n \\
2\left(G_{I J}\right)_{i j} & :=\delta_{I, n+1+i} \delta_{J, j}+\delta_{I, n+1+j} \delta_{J, i} \\
\left(H_{I J}\right)_{i j} & :=\delta_{I, i} \delta_{J, j}-\delta_{I, n+1+j} \delta_{J, n+1+i} \\
\left(P_{I J}\right)_{i j} & :=\delta_{I, n+1} \delta_{J, j}-\delta_{I, n+1+i} \delta_{J, 2 n+2} \\
\left(Q_{I J}\right)_{i j} & :=\delta_{I, i} \delta_{J, 2 n+2}+\delta_{I, n+1} \delta_{J, n+1+i} \\
R_{I J} & :=\delta_{I, n+1} \delta_{J, 2 n+2}
\end{aligned}
$$

With the conventions (4.2), (4.3), the first three matrices in (4.5) can be written down as

$$
\begin{aligned}
2 F_{i j} & :=\left(\begin{array}{cccc}
0 & 0 & E_{i j}+E_{j i} & 0 \\
0 & 0 & 0 & 0 \\
0 & 0 & 0 & 0 \\
0 & 0 & 0 & 0
\end{array}\right), i, j=1, \ldots, n \\
2 G_{i j} & :=\left(\begin{array}{ccccc}
0 & 0 & 0 & 0 \\
0 & 0 & 0 & 0 \\
E_{i j}+E_{j i} & 0 & 0 & 0 \\
0 & 0 & 0 & 0
\end{array}\right) \\
H_{i j} & :=\left(\begin{array}{cccc}
E_{i j} & 0 & 0 & 0 \\
0 & 0 & 0 & 0 \\
0 & 0 & -E_{j i} & 0 \\
0 & 0 & 0 & 0
\end{array}\right)
\end{aligned}
$$

while the matrices $P_{p}, Q_{q}, p, q=1, \ldots, n, R$ have been already defined in (2.3). 
An element $X \in \mathfrak{g}_{n}^{J}(\mathbb{R})$ can be written as matrix of $\operatorname{Sp}(n+1, \mathbb{R})$ in the base (4.6) as

$$
\begin{aligned}
X & =\sum_{i, j=1}^{n} a_{i j} H_{i j}+2 \sum_{1 \leq i<j \leq n}\left(b_{i j} F_{i j}+c_{i j} G_{i j}\right) \\
& +\sum_{1 \leq i=j \leq n}\left(b_{i j} F_{i j}+c_{i j} G_{i j}\right)+\sum_{i=1}^{n}\left(p_{i} P_{i}+q_{i} Q_{i}\right)+r R, b=b^{t}, c=c^{t} .
\end{aligned}
$$

It can be verified that

Lemma 7. The commutation relations of the generators (4.6) of the Jacobi algebra $\mathfrak{g}_{n}^{J}(\mathbb{R})$ are

$$
\begin{aligned}
{\left[H_{k l}, F_{i j}\right] } & =\delta_{l j} F_{i k}+\delta_{l i} F_{k j}, \\
{\left[G_{i j}, H_{k l}\right] } & =\delta_{k i} G_{l j}+\delta_{k j} G_{l i}, \\
4\left[F_{i j}, G_{k l}\right] & =\delta_{l i} H_{k j}+\delta_{j l} H_{i k}+\delta_{j k} H_{i l}+\delta_{i k} H_{j l}, \\
{\left[P_{p}, Q_{q}\right] } & =2 \delta_{p q} R \\
2\left[P_{p}, F_{i j}\right] & =\delta_{p i} Q_{j}+\delta_{p j} Q_{i}, \\
2\left[Q_{q}, G_{i j}\right] & =\delta_{i q} P_{j}+\delta_{j q} P_{i}, \\
{\left[P_{p}, H_{i j}\right] } & =\delta_{p i} P_{j}, \\
{\left[H_{i j}, Q_{q}\right] } & =\delta_{j q} Q_{i} .
\end{aligned}
$$

The commutation relations (4.7) of the generators of $G_{n}^{J}(\mathbb{R})$ represent the generalization of the corresponding commutation relations $(3,4),(5.1)$ and $(8.20)$ of the generators of $G_{1}^{J}(\mathbb{R})$ in [15].

4.3. The action. Following [11, §5], let us consider the restricted real group $G_{n}^{J}(\mathbb{R})_{0}$ consisting of elements of the form defined in (4.1), but $g=(M, X)$, where $X=(\lambda, \mu)$.

We consider the Siegel-Jacobi upper half space $X_{n}$ realised as in (3.11).

We introduce for $X_{n}^{J}$ the analog of parametrization used in [21, p 7], [23, p. 11], [46, $\S 38]$ for $X_{1}^{J}$

$$
u:=p v+q, v:=x+\mathrm{i} y, v=v^{t}, y>0, p, q \in M(1, n, \mathbb{R}) .
$$

It should be noted that there is an isomorphism $G_{n}^{J}(\mathbb{R}) \ni(M, X, K) \rightarrow(M, X) \in$ $G_{n}^{J}(\mathbb{R})_{0}$ through which the action of $G_{n}^{J}(\mathbb{R})_{0}$ on $X_{n}^{J}$ can be defined as in [11, Proposition $2]$.

It is easy to prove that

Lemma 8. a) If $X_{n} \ni v=x+\mathrm{i} y$, then the action of $G_{n}^{J}(\mathbb{R})_{0}$ on $X_{n}^{J}:(M, X) \times\left(v^{\prime}, u^{\prime}\right) \rightarrow$ $\left(v_{1}, u_{1}\right)$, where $M \in \operatorname{Sp}(n, \mathbb{R})$ has the expression (3.2), is given by the formulas

$$
\begin{aligned}
& v_{1}=\left(a v^{\prime}+b\right)\left(c v^{\prime}+d\right)^{-1}=\left(v^{\prime} c^{t}+d^{t}\right)^{-1}\left(v^{\prime} a^{t}+b^{t}\right), \\
& u_{1}=\left(u^{\prime}+\lambda v^{\prime}+\mu\right)\left(c v^{\prime}+d\right)^{-1} .
\end{aligned}
$$

If the modified pre-Iwasawa decomposition (3.19) is used, $v_{1}$ in (4.9a) has the equivalent expressions (3.20), (3.22) via the identification (3.23). 
b) For $\lambda, \mu \in M(1, n, \mathbb{R})$, let us consider $(p, q)$ such that

$$
\begin{aligned}
(p, q) & =(\lambda, \mu) M^{-1}=\left(\lambda d^{t}-\mu c^{t},-\lambda b^{t}+\mu a^{t}\right), \\
(\lambda, \mu) & =(p, q) M=(p a+q c, p b+q d), p, q, \lambda, \mu \in M(1, n, \mathbb{R}) .
\end{aligned}
$$

Then the action of $G_{n}^{J}(\mathbb{R})_{0}$ on $X_{n}^{J}:(M, X) \times\left(x^{\prime}, y^{\prime}, p^{\prime}, q^{\prime}\right) \rightarrow\left(x_{1}, y_{1}, p_{1}, q_{1}\right)$ is given by (4.9a), while

$$
\left(p_{1}, q_{1}\right)=(p, q)+\left(p^{\prime}, q^{\prime}\right)\left(\begin{array}{ll}
a & b \\
c & d
\end{array}\right)^{-1}=\left(p+p^{\prime} d^{t}-q^{\prime} c^{t}, q-p^{\prime} b^{t}+q^{\prime} a^{t}\right) .
$$

c) The action of $G_{n}^{J}(\mathbb{R})$ on $\tilde{X}_{n}^{J} \approx X_{n}^{J} \times \mathbb{R}$ :

$$
\begin{aligned}
(M,(\lambda, \mu), \kappa) \times\left(v^{\prime}, u^{\prime}, \kappa^{\prime}\right) & \rightarrow\left(v_{1}, u_{1}, \kappa_{1}\right), \\
(M,(\lambda, \mu), \kappa) \times\left(x^{\prime}, y^{\prime}, p^{\prime}, q^{\prime}, \kappa^{\prime}\right) & \rightarrow\left(x_{1}, y_{1}, p_{1}, q_{1}, \kappa_{1}\right)
\end{aligned}
$$

is given by (4.9), (4.11) and

$$
\kappa_{1}=\kappa+\kappa^{\prime}+\lambda q^{\prime t}-\mu p^{\prime t} .
$$

d) The 1-form

$$
\lambda^{R}=\mathrm{d} \kappa-p \mathrm{~d} q^{t}+q \mathrm{~d} p^{t}
$$

is invariant to the action (4.12) of $G_{n}^{J}(\mathbb{R})$ on $\tilde{x}_{n}^{J}$.

e) The action of $G_{n}^{J}(\mathbb{R})$ on $G_{n}^{J}(\mathbb{R})$

$$
(M,(\lambda, \mu), \kappa) \times\left(S_{n}\right)^{\prime} \rightarrow\left(S_{n}\right)_{1},
$$

is given in (3.21) for $X^{\prime}, Y^{\prime}$, while the other actions are given in a)-d) of the present Lemma.

4.4. Fundamental vector fields on $X_{n}^{J}$ and $\tilde{X}_{n}^{J}$. We calculate FVF associated to the generators of the Jacobi group on homogenous manifolds attached to $G_{n}^{J}(\mathbb{R})$.

For a symmetric matrix $x \in M(n)$ we introduce the notation

$$
\partial_{x}:=\left(\left(2-\delta_{i j}\right) \frac{\partial}{\partial x_{i j}}\right)_{i, j=1, \ldots, n} .
$$

If $a=\left(a_{1}, \ldots, a_{n}\right), b=\left(b_{1}, \ldots, b_{n}\right)$ are two $n$-vectors, we introduce also the notation

$$
(a \odot b)_{i j}:=a_{i} b_{j}+a_{j} b_{i}-a_{i} b_{j} \delta_{i j}, \quad i j=1, \ldots, n .
$$

Note the isomorphism of the representations (3.8) and (4.6) of $\mathfrak{s p}(n, \mathbb{R})$. To a matrix $A$ as in (4.6), let us denote by $\hat{A}$ the corresponding matrix in the representation (3.8).

We make the following

Remark 9. Let $z \in M(n)$. Then we have the relation (4.16)

$$
\frac{\partial}{\partial z} \mathrm{~d} z=\mathbb{1}_{n}, \text { i.e. } \frac{\partial z_{i j}}{\partial z_{p q}}=\delta_{i p} \delta_{j q} .
$$

If the matrix $z$ is symmetric, instead of (4.16) we have (4.17)

$$
D_{z} \mathrm{~d} z=\mathbb{1}_{n}, z=z^{t} \text {, i.e. }\left(D_{z}\right)_{\mu \nu} \mathrm{d} z_{\nu \chi}=\delta_{\mu \chi}, z_{\mu \nu}=z_{\nu \mu},
$$


where

$$
\left(D_{z}\right)_{\mu \nu}:=e_{\mu \nu} \frac{\partial}{\partial z_{\mu \nu}}, e_{\mu \nu}:=\frac{1+\delta_{\mu \nu}}{2}, \text { no summation! }
$$

Proof. (4.16) is evident.

Using equation [11, (4.5)] which says that for a symmetric matrix $w$ we have

$$
\frac{\partial w_{i j}}{\partial w_{p q}}=\delta_{i p} \delta_{j q}+\delta_{i q} \delta_{j p}-\delta_{i j} \delta_{p q} \delta_{i p}, w_{i j}=w_{j i},
$$

(4.17) it is verified, where the symbol $D$ in (4.18) was introdced in [11, (3.39)].

We obtain the following representations of the FVF associated to the base (4.6), (2.3) of the Lie algebra $\mathfrak{g}_{n}^{J}(\mathbb{R})$

Proposition 1. a) The fundamental vector fields in the coordinates $(v, u)$ of $X_{n}^{J}$ on which $G_{n}^{J}(\mathbb{R})$ acts as in Lemma 8 a) are given by the holomorphic FVF

$$
\begin{aligned}
F_{i j}^{*} & =\hat{F}_{i j} \frac{\partial}{\partial v}, \quad i, j=1, \ldots, n ; \\
G_{i j}^{*} & =-v \hat{G}_{i j} v \frac{\partial}{\partial v}-\left(\frac{\partial}{\partial u}\right)^{t} u \hat{G}_{i j} v ; \\
H_{i j}^{*} & =\left(\hat{E}_{i j} v+v \hat{E}_{j i}\right) \frac{\partial}{\partial v}+\left(\frac{\partial}{\partial u}\right)^{t} u \hat{E}_{j i} ; \\
P_{p}^{*} & =\hat{E}_{p} v\left(\frac{\partial}{\partial u}\right)^{t} ; Q_{q}^{*}=\hat{E}_{q}\left(\frac{\partial}{\partial u}\right)^{t} ; R^{*}=0, \quad p, q=1, \ldots, n .
\end{aligned}
$$

b) The real holomorphic FVF associated to (4.20) in the variables $(x, y, \xi, \rho)$ on $X_{n}^{J}$, where $v:=x+\mathrm{i} y, y>0, u:=\xi+\mathrm{i} \rho$ as in (4.8), are

where

$$
\begin{aligned}
F_{i j}^{*} & =\left(F_{1}^{*}\right)_{i j}, \\
G_{i j}^{*} & =\left(G_{1}^{*}\right)_{i j}+\left(\frac{\partial}{\partial \xi}\right)^{t}\left(\rho \hat{G}_{i j} y-\xi \hat{G}_{i j} x\right)-\left(\frac{\partial}{\partial \rho}\right)^{t}\left(\xi \hat{G}_{i j} y+\rho \hat{G}_{i j} x\right), \\
H_{i j}^{*} & =\left(H_{1}^{*}\right)_{i j}+\left(\frac{\partial}{\partial \xi}\right)^{t} \xi \hat{E}_{j i}+\left(\frac{\partial}{\partial \rho}\right)^{t} \rho \hat{E}_{i j}, \\
P_{p}^{*} & =\hat{E}_{p} x\left(\frac{\partial}{\partial \xi}\right)^{t}+\hat{E}_{p} y\left(\frac{\partial}{\partial \rho}\right)^{t} ; Q_{q}^{*}=\hat{E}_{q}\left(\frac{\partial}{\partial \xi}\right)^{t}, R^{*}=0,
\end{aligned}
$$

are FVF associated with the generators of $\mathfrak{s p}(n, \mathbb{R})$ corresponding to the action (4.9a) of $\operatorname{Sp}(n, \mathbb{R})$ on $X_{n}$. 
c) The FVF (4.21) in the variables $(x, y, p, q)$ on $X_{n}^{J}$, where

$$
\begin{gathered}
v=x+\mathrm{i} y, u=p v+q=\xi+\mathrm{i} \rho, \\
p=\rho y^{-1}, q=\xi-\rho y^{-1} x,
\end{gathered}
$$

are

$$
\begin{aligned}
\left(F^{*}\right)_{i j}= & \hat{F}_{i j}\left(\frac{\partial}{\partial x}-\frac{\partial}{\partial q} \odot p\right), \\
\left(G^{*}\right)_{i j}= & \left(G_{1}^{*}\right)_{i j}-\left(\left(\frac{\partial}{\partial p}\right)^{t} p\right) y^{-1} \beta+\beta\left(\frac{\partial}{\partial p} y^{-1}\right) \odot p-\alpha \frac{\partial}{\partial q} \odot p+\left(\left(\frac{\partial}{\partial q}\right)^{t} p\right) \alpha \\
& -\beta\left(\frac{\partial}{\partial q} x y^{-1}\right) \odot p+\left(\left(\frac{\partial}{\partial q}\right)^{t} p\right)\left(y^{-1} x \beta\right) \\
& -\left(\left(\frac{\partial}{\partial p}\right)^{t} q\right) y^{-1} \hat{G}_{i j} y+\left(\left(\frac{\partial}{\partial q}\right)^{t} q\right) y^{-1} \alpha, \\
\left(H^{*}\right)_{i j}= & \left(H_{1}^{*}\right)_{i j}+\left(\hat{E}_{i j} y+y \hat{E}_{j i}\right)\left[-\left(\frac{\partial}{\partial p} y^{-1}\right) \odot p+\left(\frac{\partial}{\partial q} x y^{-1}\right) \odot p\right] \\
& -\left(\hat{E}_{i j} x+x \hat{E}_{j i}\right) \frac{\partial}{\partial q} \odot p+\left(\left(\frac{\partial}{\partial q}\right)^{t} p\right)\left(x \hat{E}_{j i}-y^{-1} x y \hat{E}_{j i}\right) \\
& +\left(\left(\frac{\partial}{\partial q}\right)^{t} q\right) \hat{E}_{j i}+\left(\left(\frac{\partial}{\partial p}\right)^{t} p\right) \hat{E}_{i j}, \\
P_{p}^{*}= & E_{p}\left(\frac{\partial}{\partial p}\right)^{t}, Q_{q}^{*}=E_{q}\left(\frac{\partial}{\partial q}\right)^{t}, R^{*}=0 .
\end{aligned}
$$

d) Now we consider the action of $G_{n}^{J}(\mathbb{R})$ on $\left(u^{\prime}, v^{\prime}, \kappa^{\prime}\right) \in \tilde{X}_{n}^{J}$ as in Lemma $\left.8 \mathrm{c}\right)$. We find for $F V F F_{i j}^{*}, G_{i j}^{*}, H_{i j}^{*}$ the expressions (4.20a), (4.20b), respectively (4.20c), while instead of (4.20d), we find

$$
P_{p}^{*}=\hat{E}_{p} v\left(\frac{\partial}{\partial u}\right)^{t}+q \partial_{\kappa} ; Q_{q}^{*}=\hat{E}_{q}\left(\frac{\partial}{\partial u}\right)^{t}-p \partial_{\kappa} ; R^{*}=\partial_{\kappa} .
$$

e) The FVF on $\tilde{X}_{n}^{J}$ in the variables $(x, y, \xi, \rho, \kappa)$ are given by (4.21a), (4.21b), respectively (4.21c) for $F_{i j}^{*}, G_{i j}^{*}, H_{i j}^{*}$, while (4.21d became

$$
P_{p}^{*}=E_{p}\left(x \frac{\partial}{\partial \xi}+y \frac{\partial}{\partial \rho}\right)+q \partial_{\kappa}, Q_{q}^{*}=E_{q} \frac{\partial}{\partial \xi}-p \partial_{\kappa}, R^{*}=\partial_{\kappa}, p=\rho y^{-1}, q=\xi-\rho y^{-1} x .
$$

f) We express the FVF $F_{i j}^{*}, G_{i j}^{*}, H_{i j}^{*}$ on $\tilde{X}_{n}^{J}$ in the variables $(x, y, p, q, \kappa)$ as in (4.24a), (4.24b), respectively (4.24c), and

$$
P_{p}^{*}=E_{p}\left(x \frac{\partial}{\partial q}-y \frac{\partial}{\partial q} x y^{-1}+y \frac{\partial}{\partial p} y^{-1}\right)+q \partial_{\kappa}, Q_{q}^{*}=E_{q} \frac{\partial}{\partial q}-p \partial_{\kappa}, R^{*}=\partial_{\kappa} .
$$

Proof. a) We apply the definition of fundamental vector fields. For $P_{p}, Q_{q}, R$ on components, we find

$$
\left(P_{p}^{*}\right)_{i}=\left(\hat{E}_{p} v\right)_{i} \frac{\partial}{\partial u_{i}},\left(Q_{q}^{*}\right)_{i}=\left(\hat{E}_{q}\right)_{i} \frac{\partial}{\partial u_{i}}, R^{*}=0,
$$

which we write as in (4.20d). 
b) In order to determine the real holomorphic FVF associated to the holomorphic FVF (4.20), let $Z$ be a holomorphic vector field on a complex $n$-dimensional manifold

$$
Z:=\sum_{i=1}^{n} Z_{j} \frac{\partial}{\partial z_{j}}, Z_{j}:=A_{j}+\mathrm{i} B_{j}, A_{j}, B_{j} \in C^{\infty}(M) .
$$

Then the real holomorphic field $X=Z+\bar{Z}$ in coordinates $\left(x_{j}, y_{j}\right), z_{j}=x_{j}+\mathrm{i} y_{j}$ is, see [15, Proposition 22 in v1] or [54, Proposition 2.11],

$$
X=\sum_{i=1}^{n} A_{j} \frac{\partial}{\partial x_{j}}+B_{j} \frac{\partial}{\partial y_{j}} .
$$

c) In order to make the change of variables $(x, y, \xi, \rho) \rightarrow(x, y, p, q)$ as in (4.23), firstly it is observed that the Jacobian of the transformation is non-zero: $\frac{\partial(x, y, \xi, \rho)}{\partial(x, y, p, q)}=-y<0$.

With formula (4.19), we get the following formulas

$$
\begin{aligned}
\frac{\partial}{\partial x_{i j}} & \rightarrow\left(2-\delta_{i j}\right) \frac{\partial}{\partial x_{i j}}-\left(p \odot \frac{\partial}{\partial q}\right)_{i j} ; \\
\frac{\partial}{\partial y_{i j}} & \rightarrow\left(2-\delta_{i j}\right) \frac{\partial}{\partial y_{i j}}-\left(\frac{\partial}{\partial p} y^{-1} \odot p\right)_{i j}+\left(\frac{\partial}{\partial q} x y^{-1} \odot p\right)_{i j} ; \\
\frac{\partial}{\partial \xi_{i}} & \rightarrow \frac{\partial}{\partial q_{i}} ; \\
\frac{\partial}{\partial \rho_{l}} & \rightarrow\left(\frac{\partial}{\partial p} y^{-1}\right)_{l}-\left(\frac{\partial}{\partial q} x y^{-1}\right)_{l} ;
\end{aligned}
$$

which can be written down in the conventions (4.14), (4.15) as

$$
\begin{aligned}
\frac{\partial}{\partial x} & =\partial_{x}-\frac{\partial}{\partial q} \odot p ; \\
\frac{\partial}{\partial y} & =\partial_{y}+\left[\left(-\frac{\partial}{\partial p}+\frac{\partial}{\partial q} x\right) y^{-1}\right] \odot p ; \\
\frac{\partial}{\partial \xi} & =\frac{\partial}{\partial q} \\
\frac{\partial}{\partial \rho} & =\frac{\partial}{\partial p} y^{-1}-\frac{\partial}{\partial q} x y^{-1} .
\end{aligned}
$$

4.5. Invariant one-forms on the Jacobi group. From (4.4), we obtain

$$
g^{-1} \mathrm{~d} g=\left(\begin{array}{cccc}
A_{11} & A_{12} & A_{13} & A_{14} \\
A_{21} & A_{22} & A_{23} & A_{24} \\
A_{31} & A_{32} & A_{33} & A_{34} \\
A_{41} & A_{42} & A_{43} & A_{44}
\end{array}\right),
$$


where

$$
\begin{aligned}
& A_{11}=d^{t} \mathrm{~d} a-b^{t} \mathrm{~d} c ; A_{12}=0 ; A_{13}=d^{t} \mathrm{~d} b-b^{t} \mathrm{~d} d ; A_{14}=d^{t} \mathrm{~d} q^{t}+b^{t} \mathrm{~d} p^{t} ; \\
& A_{21}=\mathrm{d} \lambda-p \mathrm{~d} a-q \mathrm{~d} c ; A_{22}=0 ; A_{23}=\mathrm{d} \mu-p \mathrm{~d} b-q \mathrm{~d} d ; A_{24}=\mathrm{d} \kappa-p \mathrm{~d} q^{t}+q \mathrm{~d} p^{t} ; \\
& A_{31}=-c^{t} \mathrm{~d} a+a^{t} \mathrm{~d} c ; A_{32}=0 ; A_{33}=-c^{t} \mathrm{~d} b+a^{t} \mathrm{~d} d ; A_{34}=-c^{t} \mathrm{~d} q^{t}-a^{t} \mathrm{~d} p^{t} ; \\
& A_{41}=A_{42}=A_{43}=A_{44}=0 .
\end{aligned}
$$

With (4.10) and (3.3), we get from (4.29) the relations

$$
A_{24}=\mathrm{d} \kappa-p \mathrm{~d} q^{t}+q \mathrm{~d} p^{t} ; A_{34}=-A_{21}^{t} ; A_{23}=A_{14}^{t} ; A_{11}=-A_{33}^{t} .
$$

With (4.28) and (4.30), we get

Lemma 10. For $g \in \mathfrak{g}_{n}^{J}(\mathbb{R})$ as in (4.4), we have in the basis (4.6), (2.3) the expression

$$
g^{-1} \mathrm{~d} g=\sum_{i, j=1}^{n}\left(\lambda^{H}\right)_{i j} H_{i j}+\sum_{1 \leq i \leq j \leq n}\left[\left(\lambda^{F}\right)_{i j} F_{i j}+\left(\lambda^{G}\right)_{i j} G_{i j}\right]+\sum_{i=1}^{n}\left[\left(\lambda^{P}\right)_{i} P_{i}+\left(\lambda^{Q}\right)_{i} Q_{i}\right]+\lambda^{R} R,
$$

where the invariant one-forms corresponding to the generators (4.6) are

$$
\begin{aligned}
& \lambda^{F}=d^{t} \mathrm{~d} b-b^{t} \mathrm{~d} d=\left(\lambda^{F}\right)^{t}, \\
& \lambda^{G}=-c^{t} \mathrm{~d} a+a^{t} \mathrm{~d} c=\left(\lambda^{G}\right)^{t}, \\
& \lambda^{H}=d^{t} \mathrm{~d} a-b^{t} \mathrm{~d} c=\mathrm{d} b^{t} c-\mathrm{d} d^{t} a=\left(\lambda^{H}\right)^{t}, \\
& \lambda^{P}=\mathrm{d} \lambda-p \mathrm{~d} a-q \mathrm{~d} c=\mathrm{d} p a+\mathrm{d} q c=\lambda^{p}-\lambda \lambda^{H}-\mu \lambda^{G}, \\
& \lambda^{Q}=\mathrm{d} q d+\mathrm{d} p b=\mathrm{d} \mu-p \mathrm{~d} b-q \mathrm{~d} d=\lambda^{q}-\lambda \lambda^{F}+\mu \lambda^{H}, \\
& \lambda^{R}=\mathrm{d} \kappa-p \mathrm{~d} q^{t}+q \mathrm{~d} p^{t}=\lambda^{r}+\lambda \lambda^{F} \lambda^{t}-\mu \lambda^{G} \mu^{t}-2 \lambda \lambda^{H} \mu^{t},
\end{aligned}
$$

and $\lambda^{p}, \lambda^{q}, \lambda^{r}$ are given by (2.5).

Let us introduce the notation

$$
L:=y^{-1} \mathrm{~d} y, \quad R:=\mathrm{d} y y^{-1}, \quad C:=y^{-1} \mathrm{~d} x y^{-1} .
$$

With Lemma 4, we rewrite the invariant one-forms (4.31) for $G_{n}^{J}(\mathbb{R})$ as

$$
\begin{aligned}
& \lambda^{F}=X^{t} \mathrm{~d} Y-Y^{t} \mathrm{~d} X+X^{t} L Y+X^{t} C X+Y^{t} R X, \\
& \lambda^{G}=-X^{t} \mathrm{~d} Y+Y^{t} \mathrm{~d} X+Y^{t} L X-Y^{t} C Y+X^{t} R Y, \\
& \lambda^{H}=X^{t} \mathrm{~d} X+Y^{t} \mathrm{~d} Y+X^{t} L X-X^{t} C Y-Y^{t} R Y, \\
& \lambda^{P}=\mathrm{d} p\left(y X-x y^{-1} Y\right)-\mathrm{d} q y^{-1} Y, \\
& \lambda^{Q}=\mathrm{d} q y^{-1} X+\mathrm{d} p\left(y Y+x y^{-1} X\right), \\
& \lambda^{R}=\mathrm{d} \kappa-\mathrm{d} q p^{t}+\mathrm{d} p q^{t} .
\end{aligned}
$$

We have also

$$
\begin{aligned}
\lambda^{F}+\lambda^{G} & =X^{t}(L+R) Y+Y^{t}(L+R) X+X^{t} C X-Y^{t} C Y, \\
\lambda^{F}-\lambda^{G} & =2\left(X^{t} \mathrm{~d} Y-Y^{t} \mathrm{~d} X\right)+2 X^{t}(L-R) Y+X^{t} C X+Y^{t} C Y .
\end{aligned}
$$


Equations (4.31) generalize to $G_{n}^{J}(\mathbb{R}), n \in \mathbb{N}$, the corresponding equations (4.4) and (5.19) in [15] for $G_{1}^{J}(\mathbb{R})$. The last expression of $\lambda^{R}$ was obtained previously in (4.13) just in analogy to [15, (5.5f)] for the Jacobi group $G_{1}^{J}(\mathbb{R})$ and the invariance of the 1 -form was verified.

We see in (4.33) that for any $n \in\{\mathbb{N}\} \backslash\{1\}, \lambda^{F}+\lambda^{G}$ does not depend on $\mathrm{d} X, \mathrm{~d} Y$, but $\lambda^{H}$ does, while in the case $n=1$ both $\lambda^{F}+\lambda^{G}$ and $\lambda^{H}$ they does not depend on $\mathrm{d} \theta$.

Indeed, in the case of $G_{1}^{J}(\mathbb{R}), X=\cos \theta, Y=\sin \theta, y \rightarrow y^{\frac{1}{2}}$, we get equations (4.11) in 15

$$
\begin{aligned}
\lambda^{F} & =\frac{\mathrm{d} x}{y} \cos ^{2} \theta+\frac{\mathrm{d} y}{2 y} \sin 2 \theta+\mathrm{d} \theta, \\
\lambda^{G} & =-\frac{\mathrm{d} x}{y} \sin ^{2} \theta+\frac{\mathrm{d} y}{2 y} \sin 2 \theta-\mathrm{d} \theta, \\
\lambda^{H} & =-\frac{\mathrm{d} x}{2 y} \sin 2 \theta+\frac{\mathrm{d} y}{2 y} \cos 2 \theta, \\
\lambda^{F}+\lambda^{G} & =\frac{\mathrm{d} x}{y} \cos 2 \theta+\frac{\mathrm{d} y}{y} \sin 2 \theta, \\
\lambda^{F}-\lambda^{G} & =\frac{\mathrm{d} x}{y}+2 \mathrm{~d} \theta, \\
2 \lambda^{H} & =-\frac{\mathrm{d} x}{y} \sin 2 \theta+\frac{\mathrm{d} y}{y} \cos 2 \theta .
\end{aligned}
$$

With the first equation (4.33), we get

$$
\begin{aligned}
\left(\lambda^{F}+\lambda^{G}\right)^{2} & =\operatorname{tr}\left[2\left(X^{t} L Y X^{t} R Y+X^{t} L Y X^{t} R Y+X^{t} L Y Y^{t} L X-X^{t} L Y Y^{t} C Y\right)\right. \\
& +X^{t} L Y Y^{t} R X+X^{t} L Y X^{t} C X \\
& +2\left(X^{t} R Y X^{t} L Y+X^{t} R Y X^{t} R Y-X^{t} R Y Y^{t} C Y\right) \\
& +X^{t} R Y Y^{t} L X+X^{t} R Y X^{t} C X \\
& +2\left(Y^{t} L X X^{t} L Y+Y^{t} L X X^{t} C X-Y^{t} L X Y^{t} C Y\right)+Y^{t} L X X^{t} L Y \\
& +2\left(Y^{t} R X X^{t} C X-Y^{t} R X Y^{t} C Y\right) \\
& \left.+Y^{t} R X X^{t} L Y+X^{t} C X Y^{t} R X+X^{t} C X Y^{t} L X\right] .
\end{aligned}
$$

With (4.32c), we get

$$
\begin{aligned}
\left(\lambda^{H}\right)^{2} & =\left(\lambda_{1}^{H}\right)^{2}+\left(\lambda_{2}^{H}\right)^{2}, \\
\left(\lambda_{1}^{H}\right)^{2} & =\operatorname{tr}\left[X^{t}(L X-C Y) X^{t} \mathrm{~d} X+X^{t} \mathrm{~d} X X^{t}(\mathrm{~d} X+L X)+Y^{t}(\mathrm{~d} Y-R Y) Y^{t} \mathrm{~d} Y\right. \\
& -Y^{t} \mathrm{~d} Y\left(Y^{t} R+X^{t} C\right) Y+X^{t} \mathrm{~d} X Y^{t}(\mathrm{~d} Y-R Y)-X^{t} \mathrm{~d} X X^{t} C Y \\
& \left.+X^{t}(L X-C Y) Y^{t} \mathrm{~d} Y+Y^{t} \mathrm{~d} Y X^{t}(\mathrm{~d} X+L X)-Y^{t} R Y X^{t} \mathrm{~d} X\right] \\
\left(\lambda_{2}^{H}\right)^{2} & =\operatorname{tr}\left\{X^{t}(L X-C Y) X^{t} L X+Y^{t} R Y\left(Y^{t} R+X^{t} C\right) Y\right. \\
& \left.+X^{t}\left[(C Y-L X) Y^{t} R-R X Y^{t} L+(C Y-L X) X^{t} C\right] Y\right\} .
\end{aligned}
$$


With the second equation (4.33), we get

$$
\begin{aligned}
\left(\lambda^{F}-\lambda^{G}\right)^{2}= & \operatorname{tr}\left\{4\left[\left(X^{t} \mathrm{~d} Y-Y^{t} \mathrm{~d} X\right)^{2}+\left(X^{t} \mathrm{~d} Y-Y^{t} \mathrm{~d} X\right)\left(X^{t} C X+Y^{t} C Y\right)\right]\right. \\
& +2 X C X^{t} Y C Y^{t}+\left(X^{t} C X\right)^{2}+\left(Y^{t} C Y\right)^{2}+\left[X^{t}(L-R) Y\right]^{2} \\
& 2\left(X^{t} C X+Y^{t} C Y\right)\left[X^{t}(L-R) Y+Y^{t}(R-L) X\right] \\
& \left.+4 X^{t}(L-R)\left(X^{t} \mathrm{~d} Y-Y^{t} \mathrm{~d} X\right)\right\} .
\end{aligned}
$$

In the case of the Jacobi group $G_{1}^{J}(\mathbb{R})$, when $X=\cos \theta, Y=\sin \theta$ and $y \rightarrow y^{1 / 2}$, (4.35), (4.37), respectively (4.36) become what is obtained from (4.34d), i.e.

$$
\begin{aligned}
\left(\lambda^{F}+\lambda^{G}\right)^{2} & =\frac{(\cos 2 \theta \mathrm{d} x)^{2}+(\sin 2 \theta \mathrm{d} y)^{2}+\sin 4 \theta \mathrm{d} x \mathrm{~d} y}{y^{2}} ; \\
\left(\lambda_{1}^{H}\right)^{2} & =0 ; \\
\left(\lambda_{2}^{H}\right)^{2} & =\frac{(\sin 2 \theta \mathrm{d} x)^{2}+(\cos 2 \theta \mathrm{d} y)^{2}-\sin 4 \theta \mathrm{d} x \mathrm{~d} y}{4 y^{2}} ; \\
\left(\lambda^{F}-\lambda^{G}\right)^{2} & =4 \mathrm{~d} \theta^{2}+4 \frac{\mathrm{d} x \mathrm{~d} \theta}{y}+\frac{\mathrm{d} x^{2}}{y^{2}} .
\end{aligned}
$$

4.6. Invariant vector fields on the Jacobi group. Once we have determined the invariant one-forms (4.31), we have to determine the invariant vector fields orthogonal to them solving the equations

$$
<\lambda^{\alpha}\left|\left(L^{\beta}\right)^{t}>=\delta_{\alpha \beta}, \alpha, \beta=F, G, H ;<\left(L^{\alpha}\right)^{t}\right| \lambda^{\beta}>=\delta_{\alpha \beta}, \alpha, \beta=P, Q, R .
$$

We find

$$
\begin{aligned}
\left(L^{F}\right)^{t} & =\left(\frac{\partial}{\partial_{b}}\right) a+\left(\frac{\partial}{\partial_{d}}\right) c \\
\left(L^{G}\right)^{t} & =\left(\frac{\partial}{\partial_{a}}+\frac{\partial}{\partial_{b}}\right) b+\left(\frac{\partial}{\partial_{c}}+\frac{\partial}{\partial_{d}}\right) d, \\
\left(L^{H}\right)^{t} & =\left(\frac{\partial}{\partial_{a}}\right) a+\left(\frac{\partial}{\partial_{b}}\right) b+\left(\frac{\partial}{\partial_{c}}\right) c+\left(\frac{\partial}{\partial_{d}}\right) d, \\
L^{P} & =\left(\frac{\partial}{\partial_{p}}\right) d-\left(\frac{\partial}{\partial_{q}}\right) b-\left(\frac{\partial}{\partial_{\kappa}}\right)(p b+q d), \\
L^{Q} & =-\left(\frac{\partial}{\partial_{p}}\right) c+\left(\frac{\partial}{\partial_{q}}\right) a+(p a+q c) \frac{\partial}{\partial_{\kappa}}, \\
L^{R} & =\frac{\partial}{\partial_{\kappa}}
\end{aligned}
$$

In order to determine the invariant vector fields orthogonal to the invariant one-forms (4.32) as in (4.38), we have to calculate the derivative of $(a, b, c, d)$ expressed as in preIwasawa decomposition (3.15), (3.16) or modified pre-Iwasawa decomposition (3.19), but this is not an easy task.

For exemple, let's take the simpler case $d=y^{-1} X$ in (3.15), then $\mathrm{d} d=-y^{-1} \mathrm{~d} y y^{-1}+$ $y^{-1} \mathrm{~d} X$. More generally, let us consider the one-forms

$$
F_{p q}:=A_{p i} \mathrm{~d} y_{i j} B_{j q}+C_{p i} \mathrm{~d} X_{i j} D_{j q}, \quad A, B, C, D \in M(n, \mathbb{R}) .
$$


We have to determine the invariant vector field

$$
f_{q r}:=M_{q m} D_{m n}(y) N_{n r}+P_{q m} D_{m n}(X) Q_{n r}
$$

such that

$$
<F_{p q} \mid f_{q r}>=\delta_{p r}
$$

The matrices $M, N, P, Q$ such that satisfy the following matrix equation

$$
\operatorname{tr}\left[(M B)(A N)+\left(C P^{t} Q\right)\right]=\mathbb{1}_{n},
$$

must be determined, which is generally a difficult problem. If we consider the expression of $d$ in (3.19a the situation is even more complicated because of the difficulties to calculate the differential of the square root of a matrix, see Appendix 7, and we abandon the task of explicitly determining the invariant vector fields orthogonal to the left invariant one-forms (4.31).

\section{INVARIANT METRICS ON HOMOGENEOUS MANIFOLDS ASSOCIATED TO $G_{n}^{J}(\mathbb{R})$}

We follow the notation in [15, (4.15), (5.21)] for the invariant one-forms on $G_{1}^{J}(\mathbb{R})$.

Proposition 2. Let us introduce the invariant one-forms on $G_{n}^{J}(\mathbb{R})$

$$
\begin{aligned}
& \lambda_{1}:=\sqrt{\alpha}\left(\lambda^{F}+\lambda^{G}\right), \lambda_{2}:=\sqrt{\alpha} \lambda^{H}, \lambda_{3}:=\sqrt{\beta}\left(\lambda^{F}-\lambda^{G}\right), \\
& \lambda_{4}:=\sqrt{\gamma} \lambda^{P}, \lambda_{5}:=\sqrt{\gamma} \lambda^{Q}, \lambda_{6}:=\sqrt{\delta} \lambda^{R}, \quad \alpha, \beta, \gamma, \delta>0,
\end{aligned}
$$

where we use the expressions (4.32) for $\lambda^{F}, \ldots, \lambda^{R}$. The composition law (4.1) in the variables $(x, y, X, Y)$ is given in Lemma 4 or in Lemma 5 , and for $p, q, \kappa$ in Lemma 8 . Let us consider the 4-parameter left invariant metric on $G_{n}^{J}(\mathbb{R})$, which coincides with metric (5.32) on $G_{1}^{J}(\mathbb{R})$ in $[15]$

$$
\mathrm{d} s_{G_{n}^{J}(\mathbb{R})}^{2}=\sum_{i=1}^{6} \lambda_{i}^{2},
$$

where the square of the invariant one-forms $\lambda_{1}, \lambda_{2}, \lambda_{3}$ in (5.2) are given in (4.35), (4.36), respectively (4.37), and the squares of $\lambda_{4}, \lambda_{5}, \lambda_{6}$ are given taking the square of (4.32d) ... (4.32e).

Depending of the values of the parametres $\alpha, \beta, \gamma, \delta$, (5.2) gives the invariant metric on the following manifolds:

(1) if $\beta, \gamma, \delta=0$ - the Siegel upper half-plane $\mathcal{X}_{n}$;

(2) if $\gamma, \delta=0, \alpha \beta \neq 0$ - the group $\operatorname{Sp}(n, \mathbb{R})$;

(3) if $\beta, \delta=0$ - the Siegel-Jacobi half space $X_{n}^{J}$;

(4) if $\beta=0$ - the extended Siegel-Jacobi extended half space $\tilde{X}_{n}^{J}$;

(5) if $\alpha \beta \gamma \delta \neq 0$ - the Jacobi group $G_{n}^{J}(\mathbb{R})$.

The invariant vector fields (4.39), orthonormal with respect the invariant one-forms (5.1) in the sense of (4.38), are orthonormal with respect to the metric (5.2).

Proposition 2] is an extension to $G_{n}^{J}(\mathbb{R}), n \in \mathbb{N}$, of [15, Theorem 1] for $G_{1}^{J}(\mathbb{R})$. However, the expressions (4.35), (4.36), (4.37) are complicated and also the invariant vector fields (4.39) are in fact not explicitly calculated due to the difficulties signaled in Section 4.6, 
Even the metric on the Siegel upper-half space given at (1) in Proposition 2 is difficult to recognize.

We give a simple expression of the invariant metric on $X_{n}^{J}$ without the invariant oneforms, using the metric determined on the Sigel-Jacobi upper half space $X_{n}^{J}$ [8, 11, 14.

5.1. Invariant metrics on $x_{n}^{J}$ and $\tilde{X}_{n}^{J}$. Below $k, 2 k \in \mathbb{N}$ indexes the holomorphic discrete series of $\operatorname{Sp}(n, \mathbb{R})$ and $\nu>0$ indexes the representations of the Heisenberg group. We reformulate for $G_{n}^{J}(\mathbb{R}), n \in \mathbb{N}$, i[3, Proposition 1] for $G_{1}^{J}(\mathbb{R})$. The starting point is [11, Proposition 3], see also [14, Theorem 3.2].

Proposition 3. a) The Kähler two-form

$$
\begin{aligned}
-\mathrm{i} \omega_{\mathcal{D}_{n}^{J}}(W, z) & =\frac{k}{2} \operatorname{tr}(B \wedge \bar{B})+\nu \operatorname{tr}\left(A^{t} \bar{M} \wedge \bar{A}\right), A(W, z):=\mathrm{d} z^{t}+\mathrm{d} W \bar{\eta}, W \in \mathcal{D}_{n}, \\
B(W) & :=M \mathrm{~d} W, \quad M:=\left(\mathbb{1}_{n}-W \bar{W}\right)^{-1}, z \in M(1, n, \mathbb{C}), \quad \eta \in M(n, 1, \mathbb{C}),
\end{aligned}
$$

is $\left(G_{n}^{J}\right)_{0}$ invariant to the action $\operatorname{Sp}(n, \mathbb{R})_{\mathbb{C}} \times \mathbb{C}^{n}:\left(W, z^{t}\right) \rightarrow\left(W_{1}, z_{1}^{t}\right)$

$$
\left(\left(\begin{array}{cc}
\mathcal{P} & \mathcal{Q} \\
\overline{\mathcal{Q}} & \overline{\mathcal{P}}
\end{array}\right), \alpha\right) \times\left(W, z^{t}\right)=\left(\left(W \mathcal{Q}^{\dagger}+\mathcal{P}^{\dagger}\right)^{-1}\left(\mathcal{Q}^{t}+W \mathcal{P}^{t}\right),\left(W \mathcal{Q}^{\dagger}+\mathcal{P}^{\dagger}\right)^{-1}\left(z^{t}+\alpha^{t}-W \alpha^{\dagger}\right)\right)
$$

where (3.3a) are verified, i.e.

$$
\mathcal{P P}^{\dagger}-\mathcal{Q Q}^{\dagger}=\mathbb{1}_{n}, \quad \mathcal{P} \mathcal{Q}^{t}=\mathcal{Q P} \mathcal{P}^{t}, \quad \mathcal{P}^{\dagger} \mathcal{P}-\mathcal{Q}^{t} \overline{\mathcal{Q}}=\mathbb{1}_{n}, \quad \mathcal{P}^{t} \overline{\mathcal{Q}}=\mathcal{Q}^{\dagger} \mathcal{P} .
$$

We have the change of variables $(W, z) \rightarrow(W, \eta)$

$$
F C: z^{t}=\eta-W \bar{\eta} ; F C^{-1}: \eta=M\left(z^{t}+W z^{\dagger}\right),
$$

and

The complex two-form

$$
A(W, z) \rightarrow A(W, \eta)=\mathrm{d} \eta-W \mathrm{~d} \bar{\eta}
$$

is not a Kähler two-form.

$$
\omega_{\mathcal{D}_{n}^{J}}(W, \eta):=F C^{*}\left(\omega_{\mathcal{D}_{n}^{J}}(W, z)\right)
$$

The symplectic two-form $\omega_{\mathcal{D}_{n}^{J}}(W, \eta)$ is invariant to the action $(g, \alpha) \times(W, \eta) \rightarrow$ $\left(W_{1}, \eta_{1}\right)$ of $\left(G_{n}^{J}\right)_{0}$ on $\mathcal{D}_{n} \times \mathbb{C}^{n}$

$$
\eta_{1}^{t}=\mathcal{P}(\eta+\alpha)^{t}+\mathcal{Q}(\eta+\alpha)^{\dagger}
$$

where $W_{1}$ is defined in (5.4) and $(\mathcal{P}, 2)$ verify (5.5).

b) Using the partial Cayley transform

$$
\begin{gathered}
\Phi^{-1}: v=\mathrm{i}\left(\mathbb{1}_{n}-W\right)^{-1}\left(\mathbb{1}_{n}+W\right) ; u^{t}=\left(\mathbb{1}_{n}-W\right)^{-1} z^{t}, W \in \mathcal{D}_{n}, v \in \mathcal{X}_{n} ; \\
\Phi: W=\left(v-\mathrm{i} \mathbb{1}_{n}\right)^{-1}\left(v+\mathrm{i} \mathbb{1}_{n}\right), z^{t}=2 \mathrm{i}\left(v+\mathrm{i} \mathbb{1}_{n}\right)^{-1} u^{t}, z, u \in M(1, n, \mathbb{C}),
\end{gathered}
$$

we obtain

$$
A(W, z)=2 \mathrm{i}\left(v+\mathrm{i} \mathbb{1}_{n}\right)^{-1} G(v, u), \quad G(v, u)=\mathrm{d} u^{t}-\mathrm{d} v(v-\bar{v})^{-1}(u-\bar{u})^{t} .
$$

The Kähler two-form on $X_{n}^{J}$ depending on two parameters, invariant to the action (4.9) of $G_{n}^{J}(\mathbb{R})_{0}$, is

$$
-\mathrm{i} \omega_{X_{n}^{J}}(v, u)=\frac{k}{2} \operatorname{tr}(H \wedge \bar{H})+\frac{2 \nu}{\mathrm{i}} \operatorname{tr}\left(G^{t} D \wedge \bar{G}\right), \quad D:=(\bar{v}-v)^{-1}, H:=D \mathrm{~d} v .
$$


We have the change of variables $F C_{1}:(v, \eta) \rightarrow(v, u)$, where

$$
\begin{aligned}
\eta & =\left(\bar{v}-\mathrm{i} \mathbb{1}_{n}\right) D\left(v-\mathrm{i} \mathbb{1}_{n}\right)\left[\left(v-\mathrm{i} \mathbb{1}_{n}\right)^{-1} u^{t}-\left(\bar{v}-\mathrm{i} \mathbb{1}_{n}\right)^{-1} u^{\dagger}\right], \\
u^{t} & =\frac{1}{2 \mathrm{i}}\left[\left(v+\mathrm{i} \mathbb{1}_{n}\right) \eta-\left(v-\mathrm{i} \mathbb{1}_{n}\right) \bar{\eta}\right] .
\end{aligned}
$$

c) If we make the change of variables (4.8), then (5.8) becomes

$$
G^{t}(v, u)=\mathrm{d} u-p \mathrm{~d} v,
$$

and

$$
G^{t}(v, u)=G^{t}(x, y, p, q)=\mathrm{d} p v+\mathrm{d} q=\mathrm{d} p(x+\mathrm{i} y)+\mathrm{d} q .
$$

d) With (5.9), (4.8) and

$$
M(1, n, \mathbb{C}) \ni \eta:=\chi+\mathrm{i} \psi, \chi, \psi \in M(1, n, \mathbb{R}),
$$

we have the change of coordinates

$$
(x, y, p, q) \rightarrow(x, y, \chi, \psi), \quad p^{t}=\psi, q^{t}=\chi,
$$

and

$$
G^{t}(v, \eta)=G^{t}(x, y, \chi, \psi)=\mathrm{d} \psi^{t} x+\mathrm{d} \chi^{t}+\mathrm{id} \psi^{t} y
$$

We obtain

$$
\eta=(q+\mathrm{i} p)^{t} ; \quad q^{t}=\frac{1}{2}(\eta+\bar{\eta}), \quad p^{t}=\frac{1}{2 \mathrm{i}}(\eta-\bar{\eta}) .
$$

Given the change if variables (4.8) and

$$
u:=\xi+\mathrm{i} \rho,
$$

we have the change of variables

$$
(x, y, \xi, \rho) \rightarrow(x, y, p, q), \quad \xi=p x+q, \quad \rho=p y,
$$

and (5.11) becomes

$$
G^{t}(v, u)=G^{t}(x, y, \xi, \rho)=\mathrm{d} \xi-\rho y^{-1} \mathrm{~d} x+\mathrm{i}\left(\mathrm{d} \rho-\rho y^{-1} \mathrm{~d} y\right) .
$$

With (4.8), (5.10) and (5.9), we have the change of coordinates

$$
(x, y, \xi, \rho) \rightarrow(x, y, \chi, \rho), \quad \xi=\psi^{t} x+\chi^{t}, \rho=\psi^{t} y .
$$

We recall that in Perelomov's approach to CS it is considered the triplet $(G, \pi, \mathfrak{H})$, where $\pi$ is a unitary, irreducible representation of the Lie group $G$ on the separable complex Hilbert space $\mathfrak{H}$ [69].

We can introduce the normalized (un-normalized) CS-vector $\underline{e}_{x}$ (respectively, $e_{z}$ ) defined in $z \in M=G / H$

$$
\underline{e}_{x}=\exp \left(\sum_{\phi \in \Delta^{+}} x_{\phi} \boldsymbol{X}_{\phi}^{+}-\bar{x}_{\phi} \boldsymbol{X}_{\phi}^{-}\right) e_{0}, \quad e_{z}=\exp \left(\sum_{\phi \in \Delta^{+}} z_{\phi} \boldsymbol{X}_{\phi}^{+}\right) e_{0},
$$

where $e_{0}$ is the extremal weight vector of the representation $\pi, \Delta^{+}$denotes the set of positive roots of the Lie algebra $\mathfrak{g}$ of $G$, and $X_{\phi}, \phi \in \Delta$ are the generators. $X_{\phi}^{+}\left(X_{\phi}^{-}\right)$ corresponds to the positive (respectively, negative) generators. See details in [4, 13, 69,

Let us denote by $F C$ the change of variables $x \rightarrow z$ in formula (5.12) such that

$$
\underline{e}_{x}=\left(e_{z}, e_{z}\right)^{-\frac{1}{2}} e_{z} ; \quad z=F C(x) .
$$


[13. Lemma 2] verifies the assertion above for CS defined on $\mathcal{D}_{1}^{J}$, see also [5, Lemma 3], [7. Lemma 6.11 and Remark 6.12]. But the same assertions are true for CS defined on $\mathcal{D}_{n}^{J}$, see [6, Lemma 7 and Comment 8] and [8, Lemma 3.6 and Remark 3.7].

Next remark generalizes [3, Remark 1] established on $G_{1}^{J}(\mathbb{R})$ to $G_{n}^{J}(\mathbb{R}), n \in \mathbb{N}$.

Remark 11. The FC-transform (5.6) relates the un-normalized CS-vector $e_{W z}$ to the normalized one $\underline{e}_{W \eta}$

$$
\underline{e}_{W \eta}=\left(e_{W z}, e_{W z}\right)^{-\frac{1}{2}} e_{W z}, \quad W \in \mathcal{D}_{n}, z, \eta^{t} \in M(1, n, \mathbb{C}),
$$

and the $S_{n}$-variables $p, q$ are related to parameter $\eta$ defined in (5.6) by the relation

$$
\eta=(q+\mathrm{i} p)^{t} \text {. }
$$

If we denote $\alpha:=\frac{k}{4}, \gamma=: \nu$ and take into consideration assertion d) in Lemma 8 , it is obtained

Theorem 1. The metric on $X_{n}^{J}, G_{n}^{J}(\mathbb{R})_{0}$-invariant to the action in Lemma 8 , has the expressions

$$
\mathrm{d} s_{x_{n}^{J}}^{2}(x, y, p, q)=\alpha \operatorname{tr}\left[\left(y^{-1} \mathrm{~d} x\right)^{2}+\left(y^{-1} \mathrm{~d} y\right)^{2}\right]
$$

$$
\mathrm{d} s_{x_{n}^{J}}^{2}(x, y, \chi, \psi)=\alpha \operatorname{tr}\left[\left(y^{-1} \mathrm{~d} x\right)^{2}+\left(y^{-1} \mathrm{~d} y\right)^{2}\right]
$$

$$
+\gamma\left[\mathrm{d} \psi^{t}\left(x y^{-1} x+y y^{-1} y\right) \mathrm{d} \psi+\mathrm{d} \chi^{t} y^{-1} \mathrm{~d} \chi+2 \mathrm{~d} \psi^{t} x y^{-1} \mathrm{~d} \chi\right]
$$

$$
\mathrm{d} s_{x_{n}^{J}}^{2}(x, y, \xi, \rho)=\alpha \operatorname{tr}\left[\left(y^{-1} \mathrm{~d} x\right)^{2}+\left(y^{-1} \mathrm{~d} y\right)^{2}\right]
$$

$$
+\gamma\left[\mathrm{d} \xi y^{-1} \mathrm{~d} \xi^{t}+\mathrm{d} \rho y^{-1} \mathrm{~d} \rho^{t}+\rho y^{-1} \mathrm{~d} x y^{-1}\left(\rho y^{-1} \mathrm{~d} x\right)^{t}\right.
$$

$$
\left.+\rho y^{-1} \mathrm{~d} y y^{-1}\left(\rho y^{-1} \mathrm{~d} y\right)^{t}-2 \rho y^{-1} \mathrm{~d} x y^{-1} \mathrm{~d} \xi^{t}-2 \rho y^{-1} \mathrm{~d} y^{-1} \mathrm{~d} \rho^{t}\right]
$$

The three parameter metric on $\tilde{X}_{n}^{J}, G_{n}^{J}(\mathbb{R})$-invariant to the action c) in Lemma 8 , is

$$
\begin{aligned}
\mathrm{d} s_{\tilde{x}_{n}^{J}}^{2}(x, y, p, q, \kappa) & =\mathrm{d} s_{X_{n}^{J}}^{2}(x, y, p, q)+\lambda_{6}^{2} \\
& =\alpha \operatorname{tr}\left[\left(y^{-1} \mathrm{~d} x\right)^{2}+\left(y^{-1} \mathrm{~d} y\right)^{2}\right] \\
& +\gamma\left[\mathrm{d} p\left(x y^{-1} x+y y^{-1} y\right) \mathrm{d} p^{t}+\mathrm{d} q y^{-1} \mathrm{~d} q^{t}+2 \mathrm{~d} p x y^{-1} \mathrm{~d} q^{t}\right] \\
& +\delta\left(\mathrm{d} \kappa-p \mathrm{~d} q^{t}+q \mathrm{~d} p^{t}\right)^{2} .
\end{aligned}
$$

Formula (5.13) $($ (5.14) $)$ is a generalisation to $X_{n}^{J}\left(\tilde{X}_{n}^{J}\right), n \in \mathbb{N}$, of equation (5.25b) (respectively, (5.30)) in [15] corresponding to $n=1$.

\section{Appendix: Other representations of the Jacobi algebra}

We remind that the Jacobi algebra $\mathfrak{g}_{n}^{J}$, also denoted $\mathfrak{s t}(n, \mathbb{R})$ by Kirillov in [48, §18.4] or $\mathfrak{t} \mathfrak{s p}(2 n+2, \mathbb{R})$ in [49], is isomorphic with the subalgebra of Weyl algebra $A_{n}$ (see also [31]) of polynomials of degree maximum 2 in the variables $p_{1}, \ldots, p_{n}, q_{1}, \ldots, q_{n}$.

In [8] we have considered complex and biboson realization of Lie algebra $\mathfrak{s p}(n, \mathbb{R})$ as $\mathfrak{s p}(n, \mathbb{R})_{\mathbb{C}}$

$$
\mathfrak{s p}(n, \mathbb{R})_{\mathbb{C}}=\left\langle\sum_{i, j=1}^{n}\left(2 a_{i j} K_{i j}^{0}+b_{i j} K_{i j}^{+}-\bar{b}_{i j} K_{i j}^{-}\right)\right\rangle,
$$


where matrices $a=(a)_{i j}, b=(b)_{i j}, i, j,=1, \ldots, n$ verify conditions $a^{\dagger}=-a, b^{t}=b$. The realization of the generators of the symplectic group in biboson operators was observed firstly in [41], 61].

The correspondence between the generators (4.6) and the generators in [87, p. 248] of $G_{n}^{J}(\mathbb{R})$ is

$$
2 H \rightarrow A+S, 4 F \rightarrow B+T, 4 G \rightarrow B-T, D^{0} \rightarrow R, D_{1 q} \rightarrow P_{q}, \hat{D}_{1 q} \rightarrow Q_{q} .
$$

The algebra $\operatorname{wsp}(2 \mathrm{~N}, \mathbb{R})$ in $[70]$, the semidirect product of $\mathfrak{s p}(n, \mathbb{R})$ and Heisenberg, is essentially the algebra in [8], except a factor 2 .

The algebra of the inhomogeneous symplectic group $\operatorname{ISp}(2, \mathbb{R})$ in $[52$ is the same as our Jacobi algebra $\mathfrak{g}_{1}^{J}$ in [7].

The Jacobi algebra $\mathfrak{g}_{n}^{J}$ of the Jacobi group $G_{n}^{J}$ is realized as two-foton algebra [93] and $G_{n}^{J}$ is embedded in $\operatorname{Sp}(n+1, \mathbb{R})_{\mathbb{C}}$ in the context of mean-field theory in Nuclear Physics [68].

\section{Appendix: Differential of square root of a Symmetric matrix}

Let us consider a matrix $A \in M(n, \mathbb{R})$ with the eigenvalues $\lambda_{1}, \ldots, \lambda_{n}$, and let $\mathbb{R} \in$ $\alpha>0$. Then there exists a unitary matrix $U$ such that

$$
A^{\alpha}=U \operatorname{dg}\left(\lambda_{1}^{\alpha}, \ldots, \lambda_{n}^{\alpha}\right) U^{\dagger}
$$

For $\alpha=1 / 2$, i.e. $A^{1 / 2} A^{1 / 2}=A$, we have

$$
\mathrm{d} A^{1 / 2} A^{1 / 2}+A^{1 / 2} \mathrm{~d} A^{1 / 2}=\mathrm{d} A .
$$

(7.1) is a particular case of the matrix Sylvester equation

$$
A X+X B=C,
$$

where $A \in M(n), B \in M(m)$ and $X, C \in M(m, n)$. Then the solution $X$ of the matrix equation (7.2) can be written as [30]

$$
\left(\mathbb{1}_{m} \otimes A+B^{t} \otimes \mathbb{1}_{n}\right) \operatorname{vec}(X)=\operatorname{vec}(C),
$$

and the solution of the differential equation (7.1) becomes

$$
\operatorname{vec}\left(\mathrm{d} A^{1 / 2}\right)=\left(\left(A^{t}\right)^{1 / 2} \oplus A^{1 / 2}\right)^{-1} \operatorname{vec}(\mathrm{d} A) .
$$

$\otimes$ denotes in (7.3), the Kroneker product, $\oplus$ in (7.4) denotes the Kronecker sum, while $\operatorname{vec}(X)$ denotes the vectorization of the matrix $X$ [30], [59].

If the matrix $A$ is symmetric and positive definite, we introduce the notation [30]

$$
\begin{gathered}
\alpha:=\operatorname{vech}(A)=L_{n} A, \quad a:=D_{n} \alpha \\
\sigma:=\operatorname{vech}\left(A^{1 / 2}\right) \quad \sigma=L_{n} A^{1 / 2}, \quad A^{1 / 2}=D_{n} \sigma
\end{gathered}
$$

where $\operatorname{vech}(X)$ denotes the half-vectorization of the matrix $X$, while $D_{n}$ and $L_{n}$ denotes the duplication, respectively elimination matrix, see [59] for definitions.

It is obtained

$$
\mathrm{d} \sigma=L_{n}\left(\left(A^{t}\right)^{1 / 2} \oplus A^{1 / 2}\right)^{-1} D_{n} \mathrm{~d} \alpha, \quad \frac{\partial \sigma}{\partial \alpha}=L_{n}\left(\left(A^{t}\right)^{1 / 2} \oplus A^{1 / 2}\right)^{-1} D_{n} .
$$


In our case of (4.32), due to (3.19), we have to replace for the symmetric positive definite matrix $y \rightarrow y^{1 / 2}$, and formula (7.4) reads

$$
\begin{aligned}
\operatorname{vec}\left(\mathrm{d} y^{1 / 2}\right) & =\left(y^{1 / 2} \oplus y^{1 / 2}\right)^{-1} \operatorname{vec}(\mathrm{d} y)=\left(y^{1 / 2} \otimes \mathbb{1}_{n}+\mathbb{1}_{n} \otimes y^{1 / 2}\right)^{-1} \operatorname{vec}(\mathrm{d} y) . \\
\operatorname{vech}\left(\mathrm{d} y^{1 / 2}\right) & =L_{n}\left(y^{1 / 2} \oplus y^{1 / 2}\right)^{-1} D_{n} \operatorname{vech}(\mathrm{d} y) \\
& =L_{n}\left(y^{1 / 2} \otimes \mathbb{1}_{n}+\mathbb{1}_{n} \otimes y^{1 / 2}\right)^{-1} D_{n} \operatorname{vech}(\mathrm{d} y) .
\end{aligned}
$$

Acknowledgements. This research was conducted in the framework of the ANCS project program PN 190601 01/2019. I am indebted to Professor Arkadiusz Jadczyk for conversations about the symplectic group in the 2014. I would like to thank Professor Jae-Hyun Yang for sending me his collected papers.

\section{REFERENCES}

[1] S.T. Ali, J.-P. Antoine, J.-P. Gazeau, Coherent states, wavelets, and their generalizations, Springer-Verlag, New York, 2000

[2] B. Arvind, N. Dutta, Mukunda, R. Simon, The real symplectic groups in quantum mechanics and optics, Pramana - J Phys 45 (1995) 471-497, arXiv:quant-ph/9509002

[3] Elena Mirela Babalic, S. Berceanu, Remarks on the geometry of the extended Siegel-Jacobi upper half-plane, arXiv:2002.04452

[4] S. Berceanu, Realization of coherent state algebras by differential operators, in Advances in Operator Algebras and Mathematical Physics, Editors F. Boca, O. Bratteli, R. Longo, H. Siedentop, The Theta Foundation, Bucharest 2005, 1-24, arXiv:math/0504053

[5] S. Berceanu, A holomorphic representation of Lie algebras semidirect sum of semisimple and Heisenberg algebras, Romanian J. Phys. 50 (2005) 81-94

[6] S. Berceanu, A holomorphic representation of the semidirect sum of symplectic and Heisenberg Lie algebras, J. Geom. Symmetry Phys. 5 (2006) 5-13

[7] S. Berceanu, A holomorphic representation of the Jacobi algebra, Rev. Math. Phys. 18 (2006) 163-199; Errata, Rev. Math. Phys. 24 (2012) 1292001, 2 pages, arXiv:math.DG/0408219

[8] S. Berceanu, A holomorphic representation of Jacobi algebra in several dimensions, in Perspectives in Operator Algebra and Mathematical Physics, Editors F.-P. Boca, R. Purice, S. Stratila, Theta Ser. Adv. Math., Vol. 8, Theta, Bucharest, 2008, 1-25, arXiv:math.DG/0604381

[9] S. Berceanu, Generalized squeezed states for the Jacobi group, in Geometric Methods in Physics, AIP Conference Proceedings Vol. 1079, Editors P. Kielanowski, A. Odzijewicz, M. Schlichenmaier, Th. Voronov, 2008, 67-75, arXiv:0812.0717

[10] S. Berceanu, The Jacobi Group and the Squeezed States - Some Comments, in Geometric Methods in Physics, AIP Conference Proceedings Vol. 1191, Editors P. Kielanowski, S.T. Ali, A. Odzijewicz, M. Schlichenmaier, Th. Voronov, 2009, 21-29, arXiv:0910.5563

[11] S. Berceanu, A convenient coordinatization of Siegel-Jacobi domains, Rev. Math. Phys. 24 (2012) 1250024, 38 pages, arXiv:1204.5610

[12] S. Berceanu, Consequences of the fundamental conjecture for the motion on the Siegel-Jacobi disk, Int. J. Geom. Methods Mod. Phys. 10 (2013) 1250076, 18 pages, arXiv:1110.5469

[13] S. Berceanu, Coherent states and geometry on the Siegel-Jacobi disk, Int. J. Geom. Methods Mod. Phys. 11 (2014) 1450035, 25 pages, arXiv:1307.4219

[14] S. Berceanu, Balanced metric and Berezin quantization on the Siegel-Jacobi ball, SIGMA 12 (2016) 064, 24 pages, arXiv:1512.00601

[15] S. Berceanu, The real Jacobi group revisited, SIGMA 15 (2019) 096, 50 pages, arXiv:1903.1072 v1, 93 pages; v2, 54 pages

[16] S. Berceanu, A. Gheorghe, On the geometry of Siegel-Jacobi domains, Int. J. Geom. Methods Mod. Phys. 8 (2011) 1783-1798, arXiv:1011.3317v1 
[17] F. A. Berezin, Quantization in complex bounded domains, Dokl. Akad. Nauk SSSR 211 (1973) $1263-1266$

[18] F. A. Berezin, Quantization, Math. USSR-Izv. 38 (1974) 1116-1175

[19] F. A. Berezin, Quantization in complex symmetric spaces, Math. USSR-Izv. 39 (1975) 363-402

[20] F. A. Berezin, The general concept of quantization, Commun. Math. Phys. 40 (1975) 153-174

[21] R. Berndt, Some differential operators in the theory of Jacobi forms, preprint IHES/M/84/10, 1984, 31 pages

[22] R. Berndt, S. Börcherer, Jacobi Forms and Discrete Series Representations of the Jacobi group, Math. Z. 204 (1990) 13-44

[23] R. Berndt, R. Schmidt, Elements of the representation theory of the Jacobi group, Progress in Mathematics, Vol. 163, Birkhäuser Verlag, Basel, 1998

[24] B. Cahen, Global parametrization of scalar holomorphic coadjoint orbits of a quasi-Hermitian Lie group Acta Univ. Palack. Olomuc. Fac. Rerum Natur. Math. 52 (2013) 35-48

[25] B. Cahen, Stratonovich-Weyl correspondence for the Jacobi group, Commun. Math. 22 (2014) 31-48

[26] M. Cahen, S. Gutt, J. Rawnsley, Quantization of Kähler manifolds I: geometric interpretation of Berezin's quantization, J. Geom. Phys. 7 (1990) 45-62

[27] M. Cahen, S. Gutt, J. Rawnsley, Quantization of Kähler manifolds. II, Trans. Math. Soc. 337 (1993) 73-98

[28] É. Cartan, La méthode du repére mobile, la théorie des groupes continus et les espaces généralisés, Actualités scientifiques et industrielles, Vol. 194, Hermann \& Cie., Paris, 1935

[29] É. Cartan, Les espaces à connexion projective, Abh. Sem. Vektor - Tensor analysis, Moskau 4 (1937) $147-173$

[30] Derivative (or differential) of symmetric square root of a matrix, Mathematics Stack Exchange: https://math.stackexchange.com/questions/540361/derivative-or-differential-of-symmetricsquare-root-of-a-matrix

[31] J. Dixmier, Sur les algébres de Weyl, Bull. Soc. Math. France 96 (1968) 209-242

[32] V.V. Dodonov, I.A. Malkin, V.I. Man'ko, Even and odd coherent states and excitations of a singular oscillator, Physica 72 (1974) 597-615

[33] S. Donaldson, Scalar curvature and projective embeddings, I, J. Diff. Geom. 59 (2001) 479-522

[34] P.D. Drummond, Z. Ficek, Editors, Quantum Squeezing, Springer, Berlin, 2004

[35] M. Eichler, D. Zagier, The theory of Jacobi forms, Progress in Mathematics, Vol. 55, Birkhäuser Boston, Inc., Boston, MA, 1985

[36] M. Engliš, Berezin quantization and reproducing kernels on complex domains, Trans. Amer. Math. Soc. 348 (1996) 411-479

[37] E.L. Evtushik (originator) Moving-frame method, in Encyclopedia of Mathematics. http://www.encyclopediaofmath.org/index.php?title=Moving-frame-method\&oldid=17828

[38] E. Freitag, Siegelsche Modulfunktionen, Springer Verlag, 1983

[39] P.J. Freitas, On the action of the symplectic group on the Siegel upper half plane, Ph.D. Thesis, University of Illinois at Chicago, 1999

[40] G.B. Folland, Harmonic analysis in phase space, Annals of Mathematics Studies, Vol. 122, Princeton Univ. Press, Princeton, NJ, 1989

[41] S. Goshen, H.J. Lipkin, A simple independent-particle system having collective properties, Ann. Phys. (N. Y.) 6 (1959) 301-309

[42] M. de Gosson, Symplectic Geometry in Quantum Mechnaics, Birkhäuser, Basel, 2006

[43] C.R. Hagen, Scale and conformal transformations in Galilean-covariant field theory, Phys. Rev. D 5 (1972) 377-388

[44] S. Helgason, Differential geometry, Lie groups and symmetric spaces, Academic Press, New York, 1978

[45] J.N. Hollenhors, Quantum limits on resonant-mass gravitational-wave detectors, Phys. Rev. D 19 (1979) 1669-1679

[46] E. Kähler, Raum-Zeit-Individuum, Rend. Accad. Naz. Sci. XL Mem. Mat. 16 (1992) 115-177 
[47] E.H. Kennard, Zur Quantenmechanik einfacher Bewegungstypen, Zeit. Phys. 44 (1927) 326-352

[48] A. Kirillov, Éléments de la théorie des représentations, Editions Mir, Moscou, 1974

[49] A.A. Kirillov, Merits and demerits of the orbit method, Bull. Amer. Math. Soc. 36 (1999) 43-73

[50] A.A. Kirillov, Lectures on the orbit method, Graduate studies in Mathematics, Vol. 64, American Mathematical Society, Providence, Rhode Island, 2004

[51] L.K. Hua, Harmonic Analysis of Functions of Several Complex Variables in the Classical Domains, Amer. Math. Soc., Providence, R.I., 1963

[52] P. Kramer, M. Saraceno, Semicoherent states and the group $\operatorname{ISp}(2, \mathbb{R})$, Physics 114A (1982) 448453

[53] S. Kobayashi, K. Nomizu, Foundations of differential geometry, Vol. I, Interscience Publishers, New York - London, 1963

[54] S. Kobayashi, K. Nomizu, Foundations of differential geometry, Vol. II, Interscience Publishers, New York - London - Sydney, 1969

[55] M.H. Lee, Theta functions on hermitian symmetric domains and Fock representations, J. Aust. Math. Soc. 74 (2003) 201-234

[56] W. Lisiecki, A classification of coherent state representations of unimodular Lie groups, Bull. Amer. Math. Soc. 25 (1991) 37-43

[57] W. Lisiecki, Coherent state representations. A survey, Rep. Math. Phys. 35 (1995) 327-358

[58] E.Y.C. Lu, New coherent states of the electromagnetic field, Lett. Nuovo. Cimento 2 (1971) 12411244

[59] H. Lütkepohl, Handbook of matrices, John Wiley \& Sons, Chikester, 1996

[60] L. Mandel, E. Wolf, Optical coherence and quantum optics, Cambridge University Press, 1995

[61] L.D. Mlodinow, N. Papanicolau, $S O(2,1)$ algebra and the large $N$ expansion in quantum mechanics, Ann. Phys. (N. Y.) 128 (1980) 314-334

[62] B.R. Mollow, R.J. Glauber, Quantum theory of parametric amplifications: I, Phys. Rev. 160 (1967) 1076-1096

[63] H. Moscovici, Coherent state representations of nilpotent Lie groups, Commun. Math. Phys. 54 (1977) 63-68

[64] H. Moscovici, A. Verona, Coherent states and square integrable representations, Ann. Inst. Henri Poincaré 29 (1978) 139-156

[65] K.-H. Neeb, Coherent states, holomorphic extensions and highest weight representations, Pacific J. Math. 174 (1996) 230-261

[66] K.-H. Neeb, Holomorphy and convexity in Lie theory, De Gruyter Expositions in Mathematics, Vol. 28, Walter de Gruyter \& Co., Berlin, 2000

[67] U. Niederer, The maximal kinematical invariance group of the harmonic oscillator, Helv. Phys. Acta 46 (1973) 191-200

[68] S. Nishiyama, J. Da Providencia, Mean-field theory based on the $\mathfrak{J a c o b i} \mathfrak{h} \mathfrak{s p}:=$ semidirect sum $\mathfrak{h}_{N} \rtimes \mathfrak{s p}(2 N, \mathbb{R})_{\mathbb{C}}$ algebra of boson operators, J. Math. Phys. 60 (2019) 081706, 22 pages, arXiv:1809.01314

[69] A.M. Perelomov, Generalized coherent states and their applications, Texts and Monographs in Physics, Springer-Verlag, Berlin, 1986

[70] C. Quesne, Vector coherent state theory of the semidirect sum Lie algebras wsp(2N, R), J. Phys. A: Gen. 23 (1990) 847-862

[71] J.H. Rawnsley, Coherent states and Kähler manifolds, Quart. J. Math. Oxford Ser. 28 (1977) 403-415

[72] I. Satake, Fock representations and Theta Functions, Ann. Math. Studies 66 (1971) 393-405

[73] I. Satake, Unitary representations of a semi-direct products of Lie groups on $\bar{\partial}$-cohomology spaces, Math. Ann. 190 (1971) 177-202

[74] I. Satake, Factors of automorphy and Fock representations, Advances in Math. 7 (1971) 83-110

[75] I. Satake, Algebraic structures of symmetric domains, Kanô Memorial Lectures, 4, Iwanami Shoten, Tokyo; Princeton University Press, Princeton, N.J., , 1980

[76] D. Serre, Matrices: theory and applications, Springer, New York, 2002 
[77] C.L. Siegel, Symplectic geometry, Academic Press, New York, 1964

[78] R. Simon, E.C.G. Sudarshan, N. Mukunda, Gaussian pure states in quantum mechanics and the symplectic group, Phys. Rev. A 37 (1988) 3028-3038

[79] S. Sivakumar, Studies on nonlinear quantum optics, J. Opt. B Quantum Semiclass. Opt. 2 (2000) R61-R75

[80] P. Stoler, Equivalence classes of minimum uncertainty packets, Phys. Rev. D 1 (1970) 3217-3219

[81] K. Takase, A note on automorphic forms, J. Reine Angew. Math. 409 (1990) 138-171

[82] T.-Y. Tam, Computing the Iwasawa decomposition of a symplectic matrix by Cholesky factorization Appl. Math. Lett. 19 (2006) 1421-1424

[83] A. Terras, Analysis on symmetric spaces and applications, II, Springer-Verlag, Berlin, 1988

[84] J.A. Wolf, Fine structure of Hermitian symmetric spaces, in Symmetric spaces (Washington Univ., St. Louis, Mo., 1969-1970), Editors W.M. Boothby, G.L. Weiss, Marcel Decker, New York, 1972, $271-357$

[85] K.B. Wolf, The Heisenberg-Weyl ring in quantum mechanics, in Group theory and its applications, Vol. 3, Editor E.M. Loebl, Academic Press, New York, 189-247, 1975

[86] J.-H. Yang, The Siegel-Jacobi operator, Abh. Math. Sem. Univ. Hamburg 63 (1993) 135-146

[87] J.-H. Yang, The method of orbits for real Lie groups, Kyungpook Math. J. 42 (2002) 199-272, arXiv:math.RT/060205

[88] J.-H. Yang, Invariant metrics and Laplacians on the Siegel-Jacobi spaces, J. Number Theory, 127 (2007) 83-102, arXiv:math.NT/0507215

[89] J.-H. Yang, A partial Cayley transform for Siegel-Jacobi disk, J. Korean Math. Soc. 45 (2008) 781-794, arXiv:math.NT/0507216

[90] J.-H. Yang, Invariant metrics and Laplacians on the Siegel-Jacobi disk, Chin. Ann. Math. 31B (2010) 85-100, arXiv:math.NT/0507217

[91] H.P. Yuen, Two-photon coherent states of the radiation field, Phys. Rev. A 13 (1976) 2226-2243

[92] C. Ziegler, Jacobi Forms of Higher Degree, Abh. Math. Sem. Univ. Hamburg 59 (1989) 191-224

[93] W.-M. Zhang, Da-H. Feng, R. Gilmore, Coherent states: theory and some applications, Rev. Mod. Phys. 62 (1990) 867-928

(Stefan Berceanu) Horia Hulubei National Institute for Physics and Nuclear Engineering, Department of Theoretical Physics, P.O.B. MG-6, 077125 Magurele, Romania

E-mail address: Berceanu@theory.nipne.ro 